\title{
Epistemic actions as resources
}

\author{
Alexandru Baltag and Bob Coecke \\ Oxford University Computing laboratory \\ baltag / coecke@comlab.ox.ac.uk
}

\author{
Mehrnoosh Sadrzadeh \\ University of Southampton \\ ms6@ecs.soton.ac.uk
}

\begin{abstract}
We provide algebraic semantics together with a sound and complete sequent calculus for information update due to epistemic actions. This semantics is flexible enough to accommodate incomplete as well as wrong information e.g. deceit. We give a purely algebraic treatment of the muddy children puzzle, which moreover extends to situations where the children are allowed to lie and cheat. Epistemic actions, that is, information exchanges between agents $A, B, \ldots \in \mathcal{A}$, are modeled as elements of a quantale, hence conceiving them as resources. Indeed, quantales are to locales what monoidal closed categories are to Cartesian closed categories, respectively providing semantics for Intuitionistic Logic, and for non-commutative Intuitionistic Linear Logic, including Lambek calculus. The quantale $(Q, \bigvee, \bullet)$ acts on an underlying $Q$-right module $(M, \bigvee)$ of epistemic propositions and facts. The epistemic content is encoded by appearance maps, one pair $f_{A}^{M}: M \rightarrow M$ and $f_{A}^{Q}: Q \rightarrow Q$ of (lax) morphisms for each agent $A \in \mathcal{A}$. By adjunction, they give rise to epistemic modalities [12], capturing the agents' knowledge on propositions and actions. The module action is epistemic update and gives rise to dynamic modalities [20] - cf. weakest preconditions. This model subsumes the crucial fragment of Baltag, Moss and Solecki's [6] dynamic epistemic logic, abstracting it in a constructive fashion while introducing resource-sensitive structure on the epistemic actions.
\end{abstract}

Keywords: Multi-agent communication, knowledge update, resource-sensitivity, quantale, Galois adjoints, dynamic epistemic logic, sequent calculus, Lambek calculus, Linear Logic.

\section{Introduction}

Consider the following well-known puzzle. After $n$ children played in the mud $k$ of them have mud on their forehead. They can of course see each other's foreheads but not their own ones. Their father initially announces "at least one of you has mud on his forehead". After that, their father asks $k-1$ times whether they know if they are themselves dirty and $k-1$ times they all simultaneously reply "no". Now the ones which have mud on their forehead will all know this — for a detailed discussion see [12].

This Muddy Children Puzzle exposes the need for a logical account of actions and agents as dynamic and epistemic resources in situations involving information exchange. Indeed, repetition of the same announcement provides new information to the agents. In particular, these dynamic resources constitute the use-only-once resources which can be modeled by linear logic [17]. We will also deal with epistemic resources: presence of agents within a context affects the validity of deductions e.g. a deduction might not be valid in the "real world" while it is valid in the world as it appears to an agents.

We provide an algebraic semantics for such situations. Its fragment restricted to actions is a quantale logic with modal operators. Recall that quantales provide semantics for non-commutative Intuitionistic Linear Logic [40], which itself traces back to Lambek calculus [27]. The fragment for propositions is a sup-lattice logic with modal operators ( $\sup =$ join complete lattice), on which the quantale acts. Boolean 
algebras with adjoint operators, called Galois Algebras, have been used as models of Computational Tree Logic (CTL) in [18]. The pair of a sup-lattices and a quantale without the modal operators has been used in concurrency $[1,34]$ and quantum logic [10]. The crucial additional epistemic modalities are (lax-)endomorphisms of the above structure, one endomorphism-pair for each agent. The sequences of the corresponding intuitionistic sequents

$$
m_{1}, \ldots, q_{1}, \ldots, A_{1}, \ldots, m_{k}, \ldots, q_{l}, \ldots, A_{n} \vdash \delta
$$

consist of different types of formulas and for example can contain propositions $m_{1}, \ldots, m_{k}$, actions $q_{1}, \ldots, q_{l}$ and agents $A_{1}, \ldots A_{n}$, which resolve into a single proposition or action $\delta$.

Our algebraic semantics and sound and complete sequent calculus further conceptualize and abstract the usual Kripke semantics and Hilbert-style axiomatic logic for such situations e.g. the dynamic epistemic logic of Baltag, Moss and Solecki [5, 6] (BMS). Applications are secure communication, where issues of privacy, secrecy and authentication of communication protocols are central, software reliability for concurrent programs, AI, where agents are to be provided with reliable tools to reason about their environment and each other's knowledge, e-commerce, where agents need to have knowledge acquisition strategies over complex networks. The standard approach to information flow in a multi-agent system has been presented in [12] but it does not present a formal description of epistemic actions and their updates. The first attempts to formalize such actions and updates were done by Gerbrandy and Groenveld [14, 15, 16] and Plaza [31], but they only studied a restricted class of these actions. A general notion of epistemic actions and updates was introduced in [5, 6]. However, in this approach there is no account of resources in the underlying logic, and more importantly, the operations of sequential composition of actions and updating are concrete constructions on Kripke structures, rather than being taken as the fundamental operations of an abstract algebraic signature. In view of the purely Boolean nature of these Kripke models it is also worth stressing that in our proof of the Muddy children puzzle we essentially only reason by adjunction, both in terms of dynamic and epistemic residuals, but not assuming the lattice of proposition to have complements nor for it to be distributive.

We proceed as follows. First we introduce the objects of our algebra, epistemic systems, and justify their axiomatic structure. We use our setting to analyze the Muddy Children Puzzle and some of its more interesting and newer variants, involving lying children or secret communication, as well as (a simplified version of) the Man-In-The-Middle (MITM) cryptographic attack. We give examples of our structure and briefly explain how models of BMS [6] are instances of it, referring the reader for details of construction to [36]. Next we introduce the sequent calculus and give a summary of the completeness proof, referring the reader for the full proof to [36]. We illustrate the use of the sequent calculus by proving a weak permutation property for our epistemic and dynamic modalities and by encoding and deriving a property of the MITM attack. We conclude with suggestions for further elaboration.

\section{The algebra of epistemic actions and epistemic propositions}

A sup-lattice $L$ is a complete lattice and a sup-homomorphism is a map between sup-lattices which preserves arbitrary joins. We denote the bottom and top of $L$ by $\perp$ and $T$ respectively, and its atoms by $\operatorname{Atm}(L)$. A sup-lattice is atomistic iff each element can be written as the supremum of the atoms below it. Every sup-homomorphism $f^{*}: L \rightarrow M$ has a right Galois adjoint $f_{*}: M \rightarrow L$, i.e.

$$
f^{*}(a) \leq b \Leftrightarrow a \leq f_{*}(b)
$$

which preserves arbitrary infima. We denote an adjoint pair by $f^{*} \dashv f_{*}$. In computational terms, the right Galois adjoint $f_{*}$ assigns weakest preconditions to its arguments, given the program $f^{*}$. 
A quantale is a sup-lattice $Q$ with a monoid structure $(Q, \bullet, 1)$ which distributes over arbitrary joins at both sides. Since for all $a \in Q$ the maps $a \bullet-: Q \rightarrow Q$ and $-\bullet a: Q \rightarrow Q$ preserve arbitrary joins they have right Galois adjoints

$$
a \bullet-\dashv a \backslash-\quad \text { and } \quad-\bullet a \dashv-/ a,
$$

explicitly given by

$$
a \backslash b:=\bigvee\{c \in Q \mid a \bullet c \leq b\} \quad \text { and } \quad b / a:=\bigvee\{c \in Q \mid c \bullet a \leq b\}
$$

A map $f: Q \rightarrow Q$ is a quantale homomorphism if it is both a sup-homomorphism and a monoidhomomorphism. It is a lax quantale homomorphism if it is a sup homomorphism and if

$$
1 \leq f(1) \quad \text { and } \quad f(a \bullet b) \leq f(a) \bullet f(b) .
$$

Examples of quantales are: the set $\sup (L)$ of all sup-endomorphisms of a complete lattice $L$ ordered pointwisely; the set of all relations from a set $X$ to itself ordered by pointwise inclusion — this quantale is isomorphic to $\sup (\mathcal{P}(X))$; the powerset of any monoid with composition extended by continuity.

Since quantales are monoidal closed categories they provide a semantics for non-commutative Multiplicative Linear Logic $[40,17,1]$ : linearity of monoidal closed categories follows by the absence (in general) of natural morphisms $\Delta_{A}: A \rightarrow A \otimes A$ and left and right projections $p_{1}: A \otimes B \rightarrow A$ and $p_{2}: B \otimes A \rightarrow A$, and hence quantales (in general) do not satisfy $a \leq a \bullet a$ nor $a \bullet b \leq a$ nor $a \bullet b \leq b$ (where now $\bullet$ is the monoidal tensor $\otimes$ ). Note that quantales have more operators than only multiplicatives, with regard to which they are not resource-sensitive, for example we have similar inequalities for the meet of the quantale, that is we have that $a \leq a \wedge a$, and also $a \wedge b \leq a$ and $a \wedge b \leq b$.

A $Q$-right module for a quantale $Q$ is a sup-lattice $M$ with a module action

$$
-\cdot-: M \times Q \rightarrow M
$$

which preserves arbitrary joins in both arguments,

$$
m \cdot 1=m \quad \text { and } \quad m \cdot\left(q_{1} \bullet q_{2}\right)=\left(m \cdot q_{1}\right) \cdot q_{2}
$$

for all $m \in M$ and all $q_{1}, q_{2} \in Q$. We have two adjoint pairs $-\cdot q \dashv[q]-$ and $m \cdot-\dashv\{m\}-$ where

$$
[q] m:=\bigvee\left\{m^{\prime} \in M \mid m^{\prime} \cdot q \leq m\right\} \quad \text { and } \quad\{m\} m^{\prime}:=\bigvee\left\{q \in Q \mid m \cdot q \leq m^{\prime}\right\}
$$

For example, a quantale $Q$ is a $Q$-right module over itself with composition as the action and a complete lattice $L$ is a $\sup (L)$-right module with function application as the action. For details on quantales, $Q$-modules and also $Q$-enrichment we refer the reader to [25, 33, 35, 37]. For applications of these in computing, linguistics and physics we refer the reader to [1, 10, 21, 27, 30, 34].

Definition 2.1 [1] A system is a pair $(M, Q)$ with $Q$ a quantale and $M$ a $Q$-right module.

Definition 2.2 A system-endomorphism $(M, Q) \stackrel{f}{\longrightarrow}(M, Q)$ is a pair $\left(f^{M}: M \rightarrow M, f^{Q}: Q \rightarrow Q\right)$ where $f^{M}$ and $f^{Q}$ are both sup-homomorphisms, and for all $m \in M$ and $q, q^{\prime} \in Q$ we have

$$
\begin{aligned}
f^{Q}\left(q \bullet q^{\prime}\right) & \leq f^{Q}(q) \bullet f^{Q}\left(q^{\prime}\right) \\
f^{M}(m \cdot q) & \leq f^{M}(m) \cdot f^{Q}(q) \\
1 & \leq f^{Q}(1) .
\end{aligned}
$$


Hence $f^{Q}$ is lax functorial and a lax quantale homomorphism ${ }^{1}$.

Definition 2.3 An epistemic system is a tuple $\left(M, Q,\left\{f_{A}\right\}_{A \in \mathcal{A}}\right)$ where $(M, Q)$ is a system and $\left\{f_{A}\right\}_{A \in \mathcal{A}}$ are system-endomorphisms. The elements of $\mathcal{A}$ are called agents, the elements of $Q$ epistemic actions and the elements of $M$ epistemic propositions. The system-endomorphisms are called appearance maps.

Epistemic Propositions. We interpret the elements of the module as epistemic propositions and their order relation $m \leq m^{\prime}$ for $m, m^{\prime} \in M$ as logical entailment $m \vdash m^{\prime}$. The epistemic proposition $f_{A}^{M}(m)$ describes how the world appears to agent $A$ : it comprises all propositions that agent $A$ believes to hold whenever $m$ holds in the 'real world'. Two extreme examples are $f_{A}^{M}(m)=\top$, which corresponds to absence of any knowledge whatsoever, and $f_{A}^{M}(m)=m$, which stands for complete knowledge. If for $m, m^{\prime} \in M$ we have $f_{A}^{M}(m)<f_{A}^{M}\left(m^{\prime}\right)$ then agent $A$ possesses strictly more (possibly incorrect) knowledge on $m$ than on $m^{\prime}$. It also follows that $f_{A}^{M}$ indeed needs to be covariantly monotone - the additional preservation of suprema will assure existence of epistemic modalities (see below). If for $m \in M$ we have $f_{A}^{M}(m)<f_{B}^{M}(m)$ agent $A$ possesses strictly more (possibly incorrect) knowledge on $m$ than agent $B$. But indeed, this knowledge is not necessarily correct! If for $m, m^{\prime} \in M$ with $m \not \leq m^{\prime}$ we have $f_{A}^{M}(m) \leq m^{\prime}$ then agent $A$ believes incorrect information to be true, e.g. due to deceit of another agent, a malfunctioning communication channel, corrupted data etc. If the module is atomistic, then the atoms can be thought of as states - cf. Kripke structures representing epistemic scenarios (see also the following section and [4] and [36]).

Knowledge and/or belief. For each agent $A \in \mathcal{A}$ let $\square_{A}^{M}$ be defined by $f_{A}^{M} \dashv \square_{A}^{M}$. By adjunction we have $m \leq \square_{A}^{M} m^{\prime}$ if and only if $f_{A}^{M}(m) \leq m^{\prime}$, that is, "when proposition $m$ holds, agent $A$ knows/believes $m^{\prime \prime}$ ". Hence $\square_{A}^{M} m$ stands for agent $A$ 's knowledge/belief on $m$. This modality indeed covers both knowledge and belief: in contexts where no wrong belief is allowed, we read it as knowledge or justified true belief, and otherwise, as justified belief. Since $\square_{A}^{M}$ is a right Galois adjoint we have $\square_{A}^{M}\left(\bigwedge_{i} m_{i}\right)=\bigwedge_{i} \square_{A}^{M} m_{i}$. Hence it preserves the empty and binary meets, and is monotone:

$$
\square_{A}^{M} \top=\top \quad \square_{A}^{M}\left(m \wedge m^{\prime}\right)=\square_{A}^{M} m \wedge \square_{A}^{M} m^{\prime} \quad \frac{m \leq m^{\prime}}{\square_{A}^{M} m \leq \square_{A}^{M} m^{\prime}} .
$$

When $M$ is a frame (= complete Heyting algebra [23]) we can internalize the partial order using the defining property of a Heyting algebra. In the special case that $Q=\{1\}$ and $A=\{*\}$ we obtain the intuitionistic modal logic IntK $\square$ of [39]. If $M$ is moreover a complete boolean algebra (e.g. the powerset of its atoms) then Kripke's axiom $\mathbf{K}$ follows i.e.

$$
\square_{A}^{M}\left(m \rightarrow m^{\prime}\right) \rightarrow\left(\square{ }_{A}^{M} m \rightarrow \square_{A}^{M} m^{\prime}\right) .
$$

Diamonds and corresponding rules arise in that case by duality. If $M$ is atomistic and the set of atoms are denoted by $S$ then to each $f_{A}^{M}$ one can assign an accessibility relation $\stackrel{A}{\rightarrow} \subseteq S \times S$ by setting

$$
s \stackrel{A}{\rightarrow} s^{\prime} \Longleftrightarrow s^{\prime} \leq f_{A}^{M}(s)
$$

It is this relation which is primitive in ordinary epistemic logics rather than appearance maps. But in our setting, in general, this accessibility relation turns out not to be reflexive, nor (anti-)symmetric, nor transitive e.g. positive introspection $\square{ }_{A}^{M} m \leq \square_{A}^{M} \square_{A}^{M} m$ does not hold in general.

\footnotetext{
${ }^{1}$ Our notion of system endomorphism also differs from the one in the literature, (e.g. [25] and categories of modules for rings) in that we consider non-trivial homomorphisms on the quantale, a so-called change of base. Explicitly, we do not have $f(m \cdot q)=f(m) \cdot q$ for a system endomorphism $f$.
} 
Epistemic actions. We interprete the elements of the quantale as epistemic actions where the order is information ordering: if for $q, q^{\prime} \in Q$ we have $q \leq q^{\prime}$ then $q^{\prime}$ is less deterministic than $q$. The suprema $\bigvee_{i} q_{i}$ in the quantale, similar as in $[1,10]$, stand for non-deterministic choice. The action $f_{A}^{Q}(q)$ captures how $q$ appears to agent $A$. The appearance maps allow to accommodate actions such as information hiding or encryption, by $q<f_{A}^{Q}(q)$, and misinformation such as lying, cheating and deceit by $q \not \leq f_{A}^{Q}(q)$. Analogously to the case of propositions, setting $f_{A}^{Q} \dashv \square_{A}^{Q}$ stands for agent A's knowledge/belief on $q$ i.e. "when action $q$ is happening, agent $A$ believes action $q$ ' to be happening". These epistemic modalities $\square_{A}^{Q}$ satisfy the same properties as $\square_{A}^{M}$. If the quantale is atomistic then its atoms can be interpreted as deterministic actions.

Sequential composition. The quantale multiplication stands for sequential composition of epistemic actions. The multiplicative unit 1 is the void epistemic action, that is, nothing happens, sometimes referred to as skip in literature (cf. [20]). We do not require $f_{A}^{Q}(1)=1$ but only $1 \leq f_{A}^{Q}(1)$ since this enables us to accommodate suspicions, cf. eq.(3). By this we mean that even when nothing is happening one could still suspect that something hidden might be happening, say $q$, resulting in $f_{A}^{Q}(1)=1 \vee q$. Suspicions are for example important for applications to protocol security, see [36] ch. 5 for an example. On the other hand requiring $1 \leq f_{A}^{Q}(1)$ imposes rationality of the agent (vs. insanity): if nothing is happening then the agent considers nothing to be happening at least as an option. This argument carries over to appearance of sequential composition, again subject to a rationality requirement, and suspicions cause laxity, cf. eq.(1):

$$
f_{A}^{Q}(q \bullet 1)=f_{A}^{Q}(q)=f_{A}^{Q}(q) \bullet 1 \leq f_{A}^{Q}(q) \bullet f_{A}^{Q}(1) .
$$

Other situations where we have a strict inequality arise when $q \bullet q^{\prime}=\perp$ and thus $f_{A}^{Q}\left(q \bullet q^{\prime}\right)=\perp$, but $f_{A}^{Q}(q) \bullet f_{A}^{Q}\left(q^{\prime}\right) \neq \perp$ again due to the fact that the agent might suspect more options then what is actually happening — for a detailed discussion and a concrete example see [36].

Epistemic updating. The action of the quantale on the module encodes the crucial notion of epistemic updating. After performing an epistemic action $q \in Q$ on an epistemic proposition $m \in M$ we obtain a new epistemic proposition $m \cdot q \in M$. Each agent updates his knowledge according to how he perceives the epistemic action, so $f_{A}^{M}(m \cdot q)$ relates to $f_{A}^{M}(m) \cdot f_{A}^{Q}(q)$. Again suspicions impose laxity, cf. eq.(2):

$$
f_{A}^{M}(m \cdot 1)=f_{A}^{M}(m)=f_{A}^{M}(m) \cdot 1 \leq f_{A}^{M}(m) \cdot f_{A}^{Q}(1),
$$

and we can have situations where an action $q$ cannot apply to a proposition $m$, that is $m \cdot q=\perp$, and thus $f_{A}^{M}(m \cdot q)=\perp$, but the appearance of the action can apply to the appearance of the proposition, that is $f_{A}^{M}(m) \cdot f_{A}^{Q}(q) \neq \perp-$ for a detailed discussion see [36]. Situations where some of the suspected alternatives yield contradiction after update yield a process of learning (or acquiring more information): the agent will eliminate his contradiction-leading views and not anymore consider them as true options.

Dynamic modalities. Since both update.-- and quantale multiplication $-\bullet-$ preserve suprema in both arguments, a range of residuals arise, namely

$$
-\cdot q \dashv[q]-\quad m \cdot-\dashv\{m\}-\quad q \bullet-\dashv q \backslash-\quad-\bullet q \dashv-/ q
$$

for each $m \in M$ and each $q \in Q$. The residual $[q]$ - is the dynamic modality of dynamic logic [20], that is, weakest precondition. We read $[q] m$ as "after program $q$ proposition $m$ holds". On the other hand, $m \cdot q$ is the strongest postcondition. The other ones are variants on these e.g. see [21]. In particular the ones with respect to sequential composition correspond to the residuals of Lambek calculus [27] and the linear implications of non-commutative Linear Logic. 
Kernel. If $m \cdot q=\perp$ then $q$ cannot be applied to $m$. We define a kernel for an action $q \in Q$ as

$$
\operatorname{Ker}(q):=\{m \in M \mid m \cdot q=\perp\},
$$

i.e. as the co-precondition of an action $q$ (= the dual to the so-called precondition of $q)$. Since

$$
\operatorname{Ker}(q)=\downarrow(\bigvee \operatorname{Ker}(q)),
$$

"not being in the precondition of $q$ " exists as a proposition in $M$ for all $q \in Q$. Also note that the kernel of each action is the weakest proposition to which the action cannot apply, that is $\operatorname{Ker}(q)=[q] \perp$.

Stable facts. Each epistemic system has a non-epistemic part, referred to as facts, being the propositions which cannot be altered by any epistemic action. Define the stabilizer of $Q$ as

$$
\operatorname{Stab}(Q):=\{\varphi \in M \mid \forall q \in Q, \varphi \cdot q \leq \varphi\} .
$$

It consists of those epistemic propositions which are stable under the epistemic actions, or equivalently, $\varphi \leq[q] \varphi$, which expresses preservation of validity of $\varphi$ : if it is true before running $q$, it will remain true afterwards. To summarize, epistemic propositions both encode actual facts and the knowledge of each agent, that is, they have both factual and epistemic content.

\section{Examples of epistemic actions and epistemic systems}

We present some examples of epistemic actions that can exist in an epistemic system $\left(M, Q,\left\{f_{A}\right\}_{A \in \mathcal{A}}\right)$.

- Public refutation of the proposition $m \in M$ is an epistemic action $q \in Q$ with $f_{A}^{Q}(q)=q$ for all $A \in \mathcal{A}$ and for which $\operatorname{Ker}(q)=\downarrow m$.

- Private refutation to a subgroup is an action that privately refutes $m$ to the subgroup $\beta$ of agents. In this case $\operatorname{Ker}(q)$ is the same as above and $f_{A}^{Q}(q)=q$ for $A \in \beta$ and $f_{A}^{Q}(q)=1$ otherwise.

- Failure test of a proposition $m$ is an action $q$ that tests when $m$ fails. It is a particular case of private refutation where $m$ is refuted to an empty set of agents. Hence we have $\operatorname{Ker}(q)=\downarrow m$ and $f_{A}^{Q}(q)=1$ for all $A \in \mathcal{A}$.

- Public announcement is also definable in our setting. However, while "being not in the precondition of $q$ " is a proposition in $M$ for all $q \in Q$, "being in the precondition of $q$ " in general isn't one. To see this consider the lattice $\{\perp \leq a, b, c \leq \top\}$ with $q$ such that $\operatorname{Ker}(q)=\{\perp, a\}$, then both $b$ and $c$ are in the precondition but $b \vee c=\top$ isn't. The reason for this is that this lattice is non-Boolean with $a$ not having a complement. Hence public announcement of the proposition $m \in M$ is an epistemic action $q \in Q$ for which $f_{A}(q)=q$ and for which $\bigvee \operatorname{Ker}(q)$ has a Boolean complement $(\bigvee \operatorname{Ker}(q))^{c}$, satisfying $(\bigvee \operatorname{Ker}(q))^{c}=m$.

- Private announcement to a subgroup can be defined analogously. 
The Muddy Children Puzzle. This puzzle, explained in the introduction, is a paradigmatic example in the standard epistemic logic literature - e.g. [12]. In the usual encodings the communication between the father and children (i.e. father's announcement and questions and the children's answers) is not part of the actual encoding. Our approach (similar to the one in [3]) does allow to encode communications and their effects on the agents' knowledge. Our algebraic setting provides us, furthermore, with a semiautomatic equational way of doing so, instead of manually drawing Kripke structures and pruning them after each round of communication, as in [3].

We encode the puzzle in an epistemic system. The set of agents $\mathcal{A}$ includes the children $C_{1}, \cdots, C_{n}$. We assume that $C_{1}, \cdots, C_{k}$ for $1 \leq k \leq n$ are dirty. The module $M$ includes all possible initial propositions $s_{\beta}$ with $\beta \subseteq \mathcal{A}$ being those children that have mud on their forehead. For example $s_{C_{1}, \cdots, C_{k}}$ expresses the "real state" in which $C_{1}, \cdots, C_{k}$ are dirty and $C_{k+1}, \cdots, C_{n}$ are clean. Since the children cannot see their own foreheads (which might either be dirty or not) we have

$$
f_{C_{i}}^{M}\left(s_{\beta}\right)=s_{\beta \backslash\left\{C_{i}\right\}} \vee s_{\beta \cup\left\{C_{i}\right\}} .
$$

Let $D_{\emptyset}$ be the fact that no child has a dirty forehead and let $D_{i}$ be the fact that the $i$ 'th child has a dirty forehead, hence we have:

$$
\left\{D_{\emptyset}\right\} \cup\left\{D_{i} \in M \mid C_{i} \in \mathcal{A}\right\} \subseteq \operatorname{Stab}(Q)
$$

For the propositions and facts we have $s_{\beta} \leq D_{i}$ for all $C_{i} \in \beta$ and $s_{\emptyset} \leq D_{\emptyset}$, which sets that each proposition satisfies the corresponding fact. Let $q \in Q$ be a round of all children's "no" answers i.e. public refutation of $\bigvee_{i=1}^{i=n} \square_{C_{i}} D_{i}$, hence $\operatorname{Ker}(q)=\downarrow \bigvee_{i=1}^{i=n} \square_{C_{i}} D_{i}$ and $f_{C_{i}}^{Q}(q)=q$ for $1 \leq i \leq n$. Let $q_{0} \in Q$ be father's announcement that at least one child has mud on his forehead i.e. $\operatorname{Ker}\left(q_{0}\right)=\downarrow D_{\emptyset}$ and $f_{C_{i}}^{Q}\left(q_{0}\right)=q_{0}$ for $1 \leq i \leq n$.

Proposition 3.1 After $k-1$ rounds of refutations, child $j$ for $1 \leq j \leq k$ knows that he is dirty i.e.

$$
s_{\left\{C_{1}, \cdots, C_{k}\right\}} \leq\left[q_{0}(\bullet q)^{(k-1)}\right] \square_{C_{j}} D_{j}
$$

where $(\bullet q)^{(k-1)}$ denotes $\bullet q \bullet \cdots \bullet q$ with $k-1$ occurrences of $q$.

Proof. We proceed by induction on the number $k$ of dirty children. If we move the dynamic modalities in eq.(4) to the left by adjunction we obtain

$$
s_{\left\{C_{1}, \cdots, C_{k}\right\}} \cdot q_{0}(\cdot q)^{(k-1)}=s_{\left\{C_{1}, \cdots, C_{k}\right\}} \cdot\left(q_{0}(\bullet q)^{(k-1)}\right) \leq \square_{C_{j}} D_{j}
$$

using the module structure. After moving the epistemic modality to the left and by the update inequality eq.(2), it suffices to prove the following inequality

$$
f_{C_{j}}^{M}\left(s_{\left\{C_{1}, \cdots, C_{k}\right\}}\right) \cdot q_{0}(\cdot q)^{(k-1)} \leq\left(s_{\left\{C_{1}, \cdots, C_{k}\right\}} \vee s_{\left\{C_{1}, \cdots, C_{k}\right\} \backslash\left\{C_{j}\right\}}\right) \cdot q_{0}(\cdot q)^{(k-1)}
$$

which is equivalent to the following by our assumption about $f_{C_{j}}^{M}$

$$
\left(s_{\left\{C_{1}, \cdots, C_{k}\right\}} \vee s_{\left\{C_{1}, \cdots, C_{k}\right\} \backslash\left\{C_{j}\right\}}\right) \cdot q_{0}(\cdot q)^{(k-1)} \leq D_{j} .
$$

By distributivity of $\vee$ over - and the definition of suprema it suffices to prove

$$
s_{\left\{C_{1}, \cdots, C_{k}\right\}} \cdot q_{0}(\cdot q)^{(k-1)} \leq D_{j} \quad \text { and } \quad s_{\left\{C_{1}, \cdots, C_{k}\right\} \backslash\left\{C_{j}\right\}} \cdot q_{0}(\cdot q)^{(k-1)} \leq D_{j} .
$$


We respectively refer to these inequalities as eq.(6l) and eq.(6r). First we show that eq.(6l) holds for all $k$. Updating both sides of $s_{\left\{c_{1}, \cdots, c_{k}\right\}} \leq D_{j}$ by $q_{0}(\cdot q)^{(k-1)}$ we get

$$
s_{\left\{C_{1}, \cdots, C_{k}\right\}} \cdot q_{0}(\cdot q)^{(k-1)} \leq D_{j} \cdot q_{0}(\cdot q)^{(k-1)} \leq D_{j}
$$

where the last inequality follows by $D_{j} \in \operatorname{Stab}(Q)$. Hence eq.(6l). Now we prove the base case $k=1$ of our induction. Eq. $(6 r)$ is $s_{\emptyset} \cdot q_{0} \leq D_{1}$ in this case, which is true since $s_{\emptyset} \leq D_{\emptyset} \in \operatorname{Ker}\left(q_{0}\right)$ so $s_{\emptyset} \cdot q_{0}=\perp$. To prove eq.(6r) we use the inductive hypothesis in terms of eq.(5). By symmetry of $\left\{C_{1}, \cdots, C_{k}\right\}$ we have

so

$$
s_{\left\{C_{1}, \cdots, C_{k}\right\} \backslash\left\{C_{j}\right\}} \cdot q_{0}(\cdot q)^{(k-2)} \leq \square_{C_{j}} D_{j} \leq \bigvee_{i=1}^{i=n} \square_{C_{i}} D_{i}
$$

$$
s_{\left\{C_{1}, \cdots, C_{k}\right\} \backslash\left\{C_{j}\right\}} \cdot q_{0}(\cdot q)^{(k-2)} \in \operatorname{Ker}(q)
$$

and hence

$$
\perp=\left(s_{\left\{C_{1}, \cdots, C_{k}\right\} \backslash\left\{C_{j}\right\}} \cdot q_{0}(\cdot q)^{(k-2)}\right) \cdot q=s_{\left\{C_{1}, \cdots, C_{k}\right\} \backslash\left\{C_{j}\right\}} \cdot q_{0}(\cdot q)^{(k-1)} \leq D_{j}
$$

i.e. eq. $(6 r)$, what completes the proof.

Analysing the dynamics of this proof we notice that in each inductive step we show that the epistemic state $s_{\left\{C_{1}, \cdots, C_{k}\right\}} \cdot q_{0}(\cdot q)^{(k-1)}$ is included in the kernel of the refutation $q-$ cf. eq.(7). This inductive update reflects the systematic update of the children's knowledge during the process. Such a dynamics is not visible in the proofs performed in static epistemic logic [12] where there is no notion of update.

This machinery not only enables us to deal with classical epistemic scenarios in a dynamic way, but it also provides us with tools to treat (for the first time) other more complicated and realistic versions of these epistemic scenarios. As examples, we encode and analyze more complex versions of the above puzzle, in which some of the children may lie, or otherwise cheat by engaging in secret communication ${ }^{2}$, as well as an example of a cryptographic attack.

Lying Children. Assume that the same $n$ children are playing in the mud and this time only one of them, say $C_{1}$, has a dirty forehead. Their father does the announcement exactly as in the classical Muddy Children Puzzle, and then asks the same question. Now before the first round of answers, the dirty child who is a perfect reasoner, follows the proof presented above and by looking around and seeing no other dirty child, concludes that he is dirty $\square_{C_{1}} D_{1}$. But instead of announcing the truth in the first round, he lies by saying that he does not know that he is dirty. This version is encoded using the same epistemic system as muddy children above with the difference that this time we set $k=1$. Let $\bar{D}_{1}$ denote the proposition that $C_{1}$ is not dirty (it belongs to the set of facts) and set $s_{\beta} \leq \bar{D}_{1}$ where $C_{1} \notin \beta$. Note that the situations in which $C_{1}$ is not dirty satisfy this fact, for example $s_{\left\{C_{1}\right\}} \leq \bar{D}_{1}$. Denote by $\bar{q}$ the first round of answers that includes child one's lying and the others' "No!" replies. The appearance of this action to $C_{1}$ is the identity since he knows that he is lying $f_{C_{1}}(\bar{q})=\bar{q}$, whereas other children who do not know that $C_{1}$ is lying think that the action $q$ in classical muddy children (truthful public refutation) is happening, that is for $1<i \leq n$ we have $f_{C_{i}}(\bar{q})=q$. The kernel of $\bar{q}$ is the downset of the proposition in which $C_{1}$ knows he is not dirty and others know that they are dirty i.e.

$$
\operatorname{Ker}(\bar{q})=\downarrow\left(\square_{C_{1}} \bar{D}_{1} \vee \bigvee_{i=2}^{n} \square_{C_{i}} D_{i}\right) .
$$

\footnotetext{
${ }^{2}$ The cheating example was done for Kripke models of BMS by one of the authors [3], while the lying example is new.
} 
Proposition 3.2 After the first child's lying and the others' negative answers in the first round, every clean child $j$ (with $1<j \leq k$ ) thinks (wrongly) that he is dirty i.e.

$$
s_{\left\{C_{1}\right\}} \leq\left[q_{0} \bullet \bar{q}\right] \square_{C_{j}} D_{j} .
$$

Proof. We proceed in the same way as above. By moving the dynamic and epistemic modalities to the left and applying the update inequality eq.(2) we obtain

$$
f_{C_{j}}\left(s_{\left\{C_{1}\right\}}\right) \cdot f_{C_{j}}\left(q_{0}\right) \cdot f_{C_{j}}(\bar{q}) \leq D_{j} .
$$

By replacing the $f_{C_{j}}$ 's with their values we get

$$
\left(s_{\left\{C_{1}\right\}} \vee s_{\left\{C_{1}, C_{j}\right\}}\right) \cdot q_{0} \cdot q \leq D_{j}
$$

and by distributivity we have to show the following two cases (the same as in the classical version above)

$$
s_{\left\{C_{1}\right\}} \cdot q_{0} \cdot q \leq D_{j} \quad \text { and } \quad s_{\left\{C_{1}, C_{j}\right\}} \cdot q_{0} \cdot q \leq D_{j} .
$$

The second case is trivial for the same reasons as classical muddy children. For the first case we use eq.(8) proved by induction above and get $s_{\left\{C_{1}\right\}} \cdot q_{0} \in \operatorname{Ker}(q)$ and hence $\perp=s_{\left\{C_{1}\right\}} \cdot q_{0} \cdot q \leq D_{j}$.

Secret Communication. As another example, consider the original $n$ and $k$ version but in which, just before the $k-1$ 'th round, all but one of the dirty children (say, all except $C_{1}$ ), "cheat" by secretly telling each other that they are in fact dirty. We denote this action as $\pi$. In the $k-1$ 'th round, all these dirty cheating children will announce that they know they are dirty (or equivalently refute that they do not know that they are dirty) where as $C_{1}$ answers as usual. We denote this mixed round of answers by $q^{\prime}$. For the encoding of these actions in epistemic systems, that is their appearance and kernels refer to [7]. Now following the same line as in the proofs above, we can show that in the $k$ 'th round the only non-cheating child $C_{1}$ will wrongly conclude that he is clean i.e.

$$
s_{C_{1}, \ldots, C_{k}} \leq\left[q_{0} \bullet(\bullet q)^{k-2} \bullet \pi \bullet q^{\prime}\right] \square_{1} \bar{D}_{1} .
$$

The proof is done similar to the above cases and is presented in detail in [7].

A cryptographic attack. This cryptographic attack is a somewhat simplified version of the man in the middle (MITM) attack which is a primary defect of public key-based systems. Two agents $A$ and $B$ share a secret key so that they can send each other encrypted messages over some communication channel. The channel is not secure: some outsider $C$ may intercept the messages or prevent them from being delivered (although he cannot read them because he does not have the key). Suppose the encryption method is publicly known but the key is secret. It is also known that $A$ is the only one who knows an important secret for example if some fact $P$ holds or not. Suppose now that $A$ sends an encrypted message to $B$ communicating the secret. $B$ gets the message and he is convinced that it must be authentic. Now both $A$ and $B$ are convinced that they share the secret and that $C$ doesn't. However suppose that $C$ notices two features of the specific encryption method: first that the shape of the encrypted message can show whether it contains a secret or it is just junk, second that without knowing the key or the content of the message he can modify the encrypted message to its opposite i.e. if it originally said $P$ holds, it will now say that $P$ does not hold. The outsider $C$ will then secretly intercept the message, change it appropriately and send it to $B$ without knowing the secret. Now $A$ and 
$B$ mistakenly believe that they share the secret, while in fact $B$ got the wrong secret instead and $C$ has succeeded to manipulate their beliefs.

We can encode this situation in an epistemic system. The agents include $\{A, B, C\}$ and we call the message in which $P$ holds $P$ and the one in which it does not hold $\bar{P}$, these are inconsistent facts so we have $P, \bar{P} \in \operatorname{Stab}(Q)$ and $P \wedge \bar{P}=\perp, P \vee \bar{P}=\top$. Let $s, t \in M$ satisfy $s \leq P$ and $t \leq \bar{P}$. The only agent that knows if $P$ holds or not is $A$ thus $f_{A}(s)=s$ and similarly $f_{A}(t)=t$. On the other hand $B$ and $C$ do not know this so $f_{B}(s)=f_{C}(s)=f_{B}(t)=f_{C}(t)=s \vee t$. The epistemic actions that correspond to the cryptographic attack are the following: $\alpha$ in which the message $P$ is intercepted, modified and sent to $B, \beta$ in which the message $\bar{P}$ is intercepted, modified and sent to $B$, $\alpha^{\prime}$ in which $A$ sends the message $P$ to $B, \beta^{\prime}$ in which $A$ sends the message $\bar{P}$ to $B$, and finally $\gamma$ which corresponds to sending a junk message. Thus $\left\{\alpha, \beta, \alpha^{\prime}, \beta^{\prime}, \gamma\right\} \subseteq Q$. In actions $\alpha$ and $\beta$ agent $C$ is uncertain about which message $P$ or $\bar{P}$ has been sent so $f_{C}(\alpha)=f_{C}(\beta)=\alpha \vee \beta$. On the other hand, agent $A$ is sure that he has sent a message (either that $P$ holds or that it doesn't) to $B$ and that $B$ has received exactly the same secret i.e. $f_{A}(\alpha)=\alpha^{\prime}$ and $f_{A}(\beta)=\beta^{\prime}$. However if $P$ has been sent, $B$ has received $\bar{P}$ so $f_{B}(\alpha)=\beta^{\prime}$ and the other way around $f_{B}(\beta)=\alpha^{\prime}$. Further $f_{A}\left(\alpha^{\prime}\right)=f_{B}\left(\alpha^{\prime}\right)=\alpha^{\prime}$ and $f_{A}\left(\beta^{\prime}\right)=f_{B}\left(\beta^{\prime}\right)=\beta^{\prime}$ and $f_{C}\left(\alpha^{\prime}\right)=f_{C}\left(\beta^{\prime}\right)=\alpha^{\prime} \vee \beta^{\prime} \vee \gamma . C$ also considers it possible that only a junk message has been sent and that is why he sees $\gamma$ while in $\alpha^{\prime}$ and $\beta^{\prime}$. If a junk message has been sent, $A$ and $B$ are sure about it $f_{A}(\gamma)=f_{B}(\gamma)=\gamma$ while $C$ is unsure if it was a junk message or $P$ or $\bar{P}$, thus $f_{C}(\gamma)=\alpha^{\prime} \vee \beta^{\prime} \vee \gamma$. The kernel of each action comprises the states which they cannot be applied to i.e. $\operatorname{Ker}(\alpha)=\operatorname{Ker}\left(\alpha^{\prime}\right)=\downarrow \bar{P}$ and $\operatorname{Ker}(\beta)=\operatorname{Ker}\left(\beta^{\prime}\right)=\downarrow P$.

The epistemic action $\alpha \vee \beta$ expresses the action of communicating the secret $P$ or $\bar{P}$ in the above scenario. Now let us update the state $s$ with the epistemic action $\alpha \vee \beta$ and show that after update, if $P$ holds, then $A$ knows that $B$ knows that $P$ holds, that is

$$
s \cdot(\alpha \vee \beta) \leq \square_{A} \square_{B} P
$$

Since this is equal to $(s \cdot \alpha) \vee(s \cdot \beta) \leq \square_{A} \square_{B} P$ and $s \leq P \in \operatorname{Ker}(\beta)$ we get $s \cdot \beta=\perp$, so it suffices to show that $s \cdot \alpha \leq \square_{A} \square_{B} P$. By adjunction $f_{B}\left(f_{A}(s \cdot \alpha)\right) \leq P$. By eq.(2) we get $f_{A}(s \cdot \alpha) \leq f_{A}(s) \cdot f_{A}(\alpha)$, and order preservation of $f_{B}$ will give us

$$
f_{B}\left(f_{A}(s \cdot \alpha)\right) \leq f_{B}\left(f_{A}(s) \cdot f_{A}(\alpha)\right) \leq f_{B}\left(f_{A}(s)\right) \cdot f_{B}\left(f_{A}(\alpha)\right) .
$$

Now it suffices to show $f_{B}\left(f_{A}(s)\right) \cdot f_{B}\left(f_{A}(\alpha)\right) \leq P$. We do that by replacing $f_{A}$ with its values and show $f_{B}(s) \cdot f_{B}\left(\alpha^{\prime}\right) \leq P$, do the same for $f_{B}$ and get $(s \vee t) \cdot \alpha^{\prime} \leq P$, hence $\left(s \cdot \alpha^{\prime}\right) \vee\left(t \cdot \alpha^{\prime}\right) \leq P$ which is equal to $\left(s \cdot \alpha^{\prime}\right) \leq P$ since $t \leq \bar{P} \in \operatorname{Ker}\left(\alpha^{\prime}\right)$. By the assumption $s \leq P$ we obtain $s \cdot \alpha^{\prime} \leq P \cdot \alpha^{\prime}$ which leads to $s \cdot \alpha^{\prime} \leq P$ because $P$ is a fact.

BMS Models as Epistemic Systems. The Kripke semantics for dynamic epistemic logic as introduced in $[6,5]$ are examples of epistemic systems by the following theorem:

Theorem 3.3 Models of BMS are epistemic systems $\left(M, Q,\left\{f_{A}\right\}_{A \in \mathcal{A}}\right)$ with the following properties

1. Both $M$ and $Q$ are completely distributive atomistic Boolean algebras.

2. If $m$ is an atom of $M$ and $q$ is an atom of $Q$ then $m \cdot q$ is either $\perp$ or an atom.

3. If $q, q^{\prime}$ are atoms of $Q$ then $q \bullet q^{\prime}$ is an atom.

4. If $m \cdot q=\perp$ then either $m=\perp$ or $q=\perp$. 
The proof goes by constructing an epistemic system given a model of BMS and is presented in detail in [36] — and is based on ideas introduced in [4]. Key is the observation that each relation $R \subseteq S \times S$ gives rise to a sup-map $f_{R}: \mathcal{P}(S) \rightarrow \mathcal{P}(S)$ i.e. we lift the accessibility relations $\rightarrow_{A}$ of the Kripke semantics of BMS to appearance maps $f_{A}$. A model of BMS consists of two Kripke structures, one for the states as usual $\left(S, \rightarrow_{A}, \mu\right)$ and one for the deterministic actions $\left(\Sigma, \rightarrow_{A}\right.$, pre $)$ where pre $: \Sigma \rightarrow \mathcal{P}(S)$ assigns to each actions a precondition. The state model acts on the action model resulting in an updated state model, via a partial cartesian product, that is the epistemic update. Action models act on themselves via a sequential composition operation. In order to construct an epistemic system, we close the set of 'states' (= deterministic actions) of the action model under sequential composition and close the set of states of the state model under update. The closure of the states yields the atoms of module and the closure of deterministic actions yields the atoms of quantale, and we get a Boolean epistemic system by taking their powersets $\left(\mathcal{P}(S), \mathcal{P}(\Sigma),\left\{f_{A}\right\}_{A \in \mathcal{A}}\right)$. Operations of this epistemic system are constructions of BMS, e.g. epistemic update and sequential composition extended pointwisely to subsets of states and actions. The epistemic and dynamic modalities arise, as before, as adjoints to the lifted appearance and update maps, but moreover and because of the boolean complementation we get a de Morgan dual for each of these modalities, in particular the de Morgan dual of the epistemic modality $\left(\square_{A}(-)^{c}\right)^{c}$ stands for the $\diamond$-modality of standard epistemic logic.

\section{The sequent calculus of epistemic systems}

We have two different sequent systems, a $Q$-system and an $M$-system, both of them are intuitionistic in the sense that they have only one formula on the right hand side of the turnstile. The $Q$-system corresponds to the quantale part of an epistemic system, that is the quantale together with its $f_{A}^{Q}$-maps, and the $M$-system corresponds to the module together with its $f_{A}^{M}$-maps.

The $Q$-system. The formulas of the $Q$-system, denoted as $L_{Q}$, are generated by the following syntax:

$$
q::=\top|\perp| \sigma|1| q \bullet q|q / q| q \backslash q|q \vee q| q \wedge q\left|f_{A}^{Q}(q)\right| \square_{A}^{Q} q
$$

where $A$ is in the set $\mathcal{A}$ of agents, and $\sigma$ is in a set $V_{Q}$ of atomic action variables. The sequents of the $Q$-system are called $Q$-sequents and are denoted as

$$
\Gamma \vdash_{Q} q
$$

where $\Gamma$ is a sequence of actions and agents, that is $\Gamma \in\left(L_{Q} \cup \mathcal{A}\right)^{*}$, and $q$ is a single action, that is $q \in L_{Q}$. To assign meaning to the sequents of the $Q$-system, we introduce

$$
-\odot_{Q}-: L_{Q} \times\left(L_{Q} \cup \mathcal{A}\right) \rightarrow L_{Q}
$$

by putting

$$
q \odot_{Q} q^{\prime}:=q \bullet q^{\prime} \quad q \odot_{Q} A:=f_{A}^{Q}(q) .
$$

For $\Gamma=\left(\gamma_{1}, \cdots, \gamma_{n}\right) \in\left(L_{Q} \cup \mathcal{A}\right)^{*}$ we take the convention

$$
\bigodot_{Q} \Gamma:=\left(\left(\left(\left(1 \odot_{Q} \gamma_{1}\right) \odot_{Q} \gamma_{2}\right) \odot_{Q} \gamma_{3}\right) \cdots\right) \odot_{Q} \gamma_{n} .
$$

As an example, the sequence $\Gamma=\left(q, A, q^{\prime}\right)$ corresponds to

$$
\bigodot_{Q} \Gamma=\left(\left(1 \odot_{Q} q\right) \odot_{Q} A\right) \odot_{Q} q^{\prime}=f_{A}^{Q}(1 \bullet q) \bullet q^{\prime}=f_{A}^{Q}(q) \bullet q^{\prime} .
$$


Adding the multiplicative unit to the beginning of the sequence will allow us to avoid non-well defined $\odot_{Q}$-expressions for sequences such as $\Gamma=A$, which will now mean $\bigodot_{Q} A=f_{A}^{Q}(1)$. Indeed, the operation $\bigodot_{Q}$ constitutes our semantic interpretation of the comma for $Q$-sequents. For simplicity we denote the semantics of a formula by the formula itself i.e. rather than $\llbracket \bigodot_{Q} \Gamma \rrbracket$ and $\llbracket q \rrbracket$ we write $\bigodot_{Q} \Gamma$ and $q$. The empty sequence on the left hand side stands for 1 and we do not allow for the empty sequence on the right hand side. We define a satisfaction relation $\models_{Q}$ on the $Q$-system as follows:

$$
\Gamma \models_{Q} q^{\prime} \Longleftrightarrow \bigodot_{Q} \Gamma \leq q^{\prime}
$$

We say that a sequent $\Gamma \vdash_{Q} q^{\prime}$ is valid if and only if $\Gamma \models_{Q} q^{\prime}$. In this way we identify any $Q$-sequence $\Gamma$ with a $Q$-formula and its corresponding element of the quantale.

Ordered monoids have first been used by Lambek to model Lambek-calculus. Yetter showed that quantales are models of non-commutative Linear Logic [40]. The extension of these systems to epistemic modalities and quantales with operators is new. So the operational and unit rules for the $Q$-system are the rules for Non-Commutative Intuitionistic Linear Logic, extended with an agent context. In order to see the connection with Linear Logic note that our multiplication $\bullet$ is the tensor of Linear Logic, our disjunction is the Linear Logic sum, the conjunction is \&, and our left and right residuals are $\circ-$ and $-\circ$. In a table:

\begin{tabular}{|c|c|}
\hline Q-system & Linear Logic \\
\hline \hline 1 & 1 \\
\hline$\top$ & $\top$ \\
\hline$\perp$ & 0 \\
\hline$\bullet$ & $\otimes$ \\
\hline$/$ & $\circ-$ \\
\hline$\backslash$ & $-\circ$ \\
\hline$\vee$ & $\oplus$ \\
\hline$\wedge$ & $\&$ \\
\hline
\end{tabular}

The axiom and unit rules of the $Q$-system are:

$$
\begin{array}{|cc|}
\hline \frac{\Gamma, \Gamma^{\prime} \vdash_{Q} q}{\Gamma, 1, \Gamma^{\prime} \vdash_{Q} q} 1 L & \overline{\vdash_{Q} 1} 1 R \\
\frac{\Gamma, \perp, \Gamma^{\prime} \vdash_{Q} q}{\Gamma L} 1 L & \frac{}{\Gamma \vdash_{Q} \top} \top R \\
\hline
\end{array}
$$

The operational rules of the $Q$-system including those on epistemic modalities are: 


\begin{tabular}{|c|c|}
\hline $\begin{array}{c}\frac{\Gamma \vdash_{Q} q}{\Gamma, A \vdash_{Q} f_{A}^{Q}(q)} f_{A}^{Q} R \\
\frac{\Gamma \vdash_{Q} \square_{A}^{Q} q}{\Gamma, A \vdash_{Q} q} \square_{A}^{Q} R \\
\frac{\Gamma, q_{1}, q_{2}, \Gamma^{\prime} \vdash_{Q} q}{\Gamma, q_{1} \bullet q_{2}, \Gamma^{\prime} \vdash_{Q} q} \bullet L \\
\frac{\Gamma, q_{1}, \Gamma^{\prime} \vdash_{Q} q \quad \Gamma, q_{2}, \Gamma^{\prime} \vdash_{Q} q}{\Gamma, q_{1} \vee q_{2}, \Gamma^{\prime} \vdash_{Q} q} q_{2}, \Gamma^{\prime} \vdash_{Q} q \\
\Gamma, q_{1}, \Gamma^{\prime} \vdash_{Q} q \\
\frac{\Gamma, q_{1} \wedge q_{2}, \Gamma^{\prime} \vdash_{Q} q}{\Gamma, q_{2}, \Gamma^{\prime} \vdash_{Q} q} \wedge L 2 \\
\frac{\Gamma_{Q} \vdash_{Q} q_{2} \quad q_{1} \vdash_{Q} q}{q_{1} / q_{2}, \Gamma_{Q} \vdash_{Q} q} / L \\
\frac{\Gamma_{Q} \vdash_{Q} q_{1} \quad q_{2} \vdash_{Q} q}{\Gamma_{Q}, q_{1} \backslash q_{2} \vdash_{Q} q} \backslash L\end{array}$ & $\begin{array}{c}\frac{\Gamma_{Q}, A, \Gamma \vdash_{Q} q}{f_{A}^{Q}\left(\Gamma_{Q}\right), \Gamma \vdash_{Q} q} f_{A}^{Q} L \\
\frac{\Gamma_{Q}, \Gamma \vdash_{Q} q}{\square_{A}^{Q}\left(\Gamma_{Q}\right), A, \Gamma \vdash_{Q} q} \square_{A}^{Q} L \\
\frac{\Gamma_{Q}, \Gamma_{A} \vdash_{Q} q_{1} \quad \Gamma_{Q}^{\prime}, \Gamma_{A} \vdash_{Q} q_{2}}{\Gamma_{Q}, \Gamma_{Q}^{\prime}, \Gamma_{A} \vdash_{Q} q_{1} \bullet q_{2}} \bullet R \\
\frac{\Gamma \vdash_{Q} q_{1}}{\Gamma \vdash_{Q} q_{1} \vee q_{2}} \vee R 1 \quad \frac{\Gamma \vdash_{Q} q_{2}}{\Gamma \vdash_{Q} q_{1} \vee q_{2}} \vee R 2 \\
\frac{\Gamma \vdash_{Q} q_{1}}{\Gamma \vdash_{Q} q_{1} \wedge q_{2} q_{2}} \wedge R \\
\frac{\Gamma, q_{2} \vdash_{Q} q_{1}}{\Gamma \vdash_{Q} q_{1} / q_{2}} / R \\
\frac{q_{1}, \Gamma_{Q} \vdash_{Q} q_{2}}{\Gamma_{Q} \vdash_{Q} q_{1} \backslash q_{2}} \backslash R\end{array}$ \\
\hline
\end{tabular}

As in non-commutative Linear Logic we have no weakening, contraction and exchange rules for actions. Our structural rules consist of the usual cut rule and a rule to encode the relation between appearance maps and the unit of composition - eq.(3) in the algebra. These two rules are:

\begin{tabular}{|c|}
\hline$\Gamma^{\prime} \vdash_{Q} q \Gamma, q, \Gamma^{\prime \prime} \vdash_{Q} q^{\prime}$ \\
$\Gamma, \Gamma^{\prime}, \Gamma^{\prime \prime} \vdash_{Q} q^{\prime}$
\end{tabular} cut $\quad \frac{A \vdash_{Q} q}{1 \vdash_{Q} q}$ Agent

The $M$-system. The formulas of the $M$-system, denoted as $L_{M}$, are generated by the following syntax:

$$
m::=\perp|\top| p|s| m \wedge m|m \vee m|[q] m|m \cdot q| \square_{A}^{M} m \mid f_{A}^{M}(m)
$$

where $A$ is in the set $\mathcal{A}$ of agents, $p$ is in the set $\Phi$ of facts, and $s$ is in a set $V_{M}$ of atomic propositional variables. The sequents of the $M$-system are called $M$-sequents and are denoted as

$$
\Gamma \vdash \vdash_{M} m
$$

where $\Gamma$ is a sequence of propositions, actions, and agents, that is $\Gamma \in\left(L_{M} \cup L_{Q} \cup \mathcal{A}\right)^{*}$ and $m$ is a single proposition, that is $m \in L_{M}$. To assign meaning to the sequents of an $M$-sequent we consider

$$
-\odot_{M}-: L_{M} \times\left(L_{M} \cup L_{Q} \cup \mathcal{A}\right) \rightarrow L_{M}
$$

now by putting

$$
m \odot_{M} A:=f_{A}^{M}(m) \quad m \odot_{M} q:=m \cdot q \quad m \odot_{M} m^{\prime}:=m \wedge m^{\prime} .
$$

For $\Gamma=\left(\gamma_{1}, \cdots, \gamma_{n}\right) \in\left(L_{M} \cup L_{Q} \cup \mathcal{A}\right)^{*}$ we take the convention

$$
\bigodot_{M} \Gamma:=\left(\left(\left(\left(\top \odot_{M} \gamma_{1}\right) \odot_{M} \gamma_{2}\right) \odot_{M} \gamma_{3}\right) \cdots\right) \odot_{M} \gamma_{n} .
$$


As an example, the sequence $\Gamma=\left(m, A, q, B, m^{\prime}\right)$ corresponds to

$$
\bigodot_{M} \Gamma=\left(\left(\left(\left(\top \odot_{M} m\right) \odot_{M} A\right) \odot_{M} q\right) \odot_{M} B\right) \odot_{M} m^{\prime}=f_{B}\left(f_{A}(\top \wedge m) \cdot q\right) \wedge m^{\prime}=f_{B}\left(f_{A}(m) \cdot q\right) \wedge m^{\prime} .
$$

Sequences of only one agent $\Gamma=A$ will mean $\bigodot_{M} \Gamma=f_{A}^{M}(\top)$ and sequences of only one action $\Gamma=q$ will mean $\bigodot_{M} \Gamma=\top \cdot q$. The empty sequence on the left hand side stands for $T$, here we also allow for an empty right hand side, which stands for $\perp$. As before, we denote the semantics of a formula by the formula itself. We define

$$
\Gamma \models{ }_{M} m^{\prime} \Longleftrightarrow \bigodot_{M} \Gamma \leq m^{\prime}
$$

and say that a sequent $\Gamma \vdash_{M} m^{\prime}$ is valid if and only if $\Gamma \models_{M} m^{\prime}$. In this way we identify any $M$-sequence $\Gamma$ with an $M$-formula and its corresponding element of the module.

The rules of the $M$-system correspond to a non-distributive lattice logic extended with an agent context for our epistemic modalities. The axiom and unit rules of the $M$-system are:

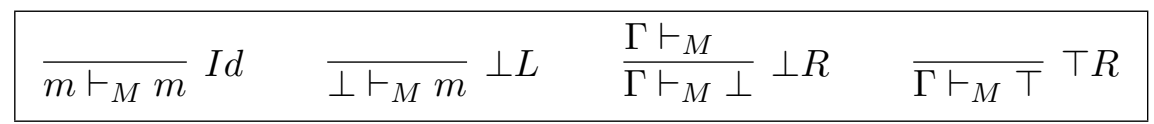

The operational rules of the $M$-system for the lattice operations and modalities are:

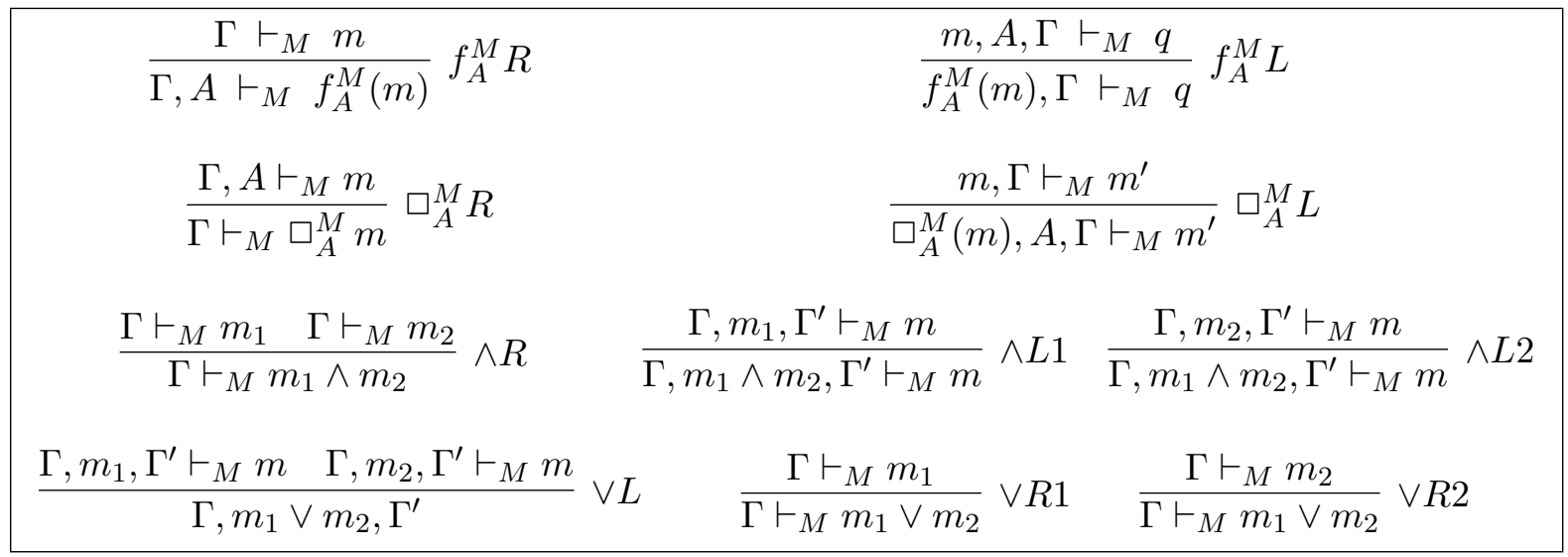

The structural rules are the usual ones augmented with a rule encoding stability of facts under update:

$$
\begin{array}{ccc}
\frac{\Gamma, \Gamma^{\prime} \vdash m}{\Gamma, m^{\prime}, \Gamma^{\prime} \vdash_{M} m} \text { weak } & \frac{\Gamma, m^{\prime}, m^{\prime}, \Gamma^{\prime} \vdash_{M} m}{\Gamma, m^{\prime}, \Gamma^{\prime} \vdash_{M} m} \text { contr } & \frac{\Gamma, m^{\prime \prime}, m^{\prime}, \Gamma^{\prime} \vdash_{M} m}{\Gamma, m^{\prime}, m^{\prime \prime}, \Gamma^{\prime} \vdash_{M} m} \text { exch } \\
\frac{\Gamma^{\prime} \vdash_{M} m^{\prime} \quad \Gamma, m^{\prime}, \Gamma^{\prime \prime} \vdash_{M} m}{\Gamma, \Gamma^{\prime}, \Gamma^{\prime \prime} \vdash_{M} m} \text { Mcut } & \frac{\Gamma \vdash_{M} p}{\Gamma, q \vdash_{M} p} \text { fact }
\end{array}
$$

The $M Q$-system. Since the core of our approach is the action of the quantale on the module, we also have mixed rules for epistemic update and dynamic modality consisting of both $M$ - and $Q$-sequents: 


$$
\begin{array}{|cc|}
\hline \frac{\Gamma, q, \Gamma^{\prime} \vdash_{M} m}{\Gamma \cdot q, \Gamma^{\prime} \vdash_{M} m} \cdot L & \frac{\Gamma, \Gamma_{A} \vdash_{M} m \quad \Gamma_{Q}, \Gamma_{A} \vdash_{Q} q}{\Gamma, \Gamma_{Q}, \Gamma_{A} \vdash_{M} m \cdot q} \cdot R \\
\frac{m^{\prime} \vdash_{M} m \quad q \vdash_{Q} \Gamma_{Q}}{[q] m^{\prime}, \Gamma_{Q} \vdash_{M} m} D y L & \frac{\Gamma, q \vdash_{M} m}{\Gamma \vdash_{M}[q] m} D y R \\
\hline
\end{array}
$$

The action of the quantale on the module preserves the unit of multiplication, is disjunction preserving in both arguments, and satisfies an associativity condition with regard to composition of actions. In order to prove the same properties for epistemic update (and dual ones for dynamic modality) in the $M$-system, we should be able to work with the quantale operations in $M$-sequents. So we have the following rules that include for example update with unit, composition, and choice of actions:

\begin{tabular}{|cc|}
\hline$\frac{\Gamma, \Gamma^{\prime} \vdash_{M} m}{\Gamma, 1, \Gamma^{\prime} \vdash_{M} m} 1 M L$ & $\frac{\Gamma, q_{1}, q_{2}, \Gamma^{\prime} \vdash_{M} m}{\Gamma, q_{1} \bullet q_{2}, \Gamma^{\prime} \vdash_{M} m} \bullet M L$ \\
$\frac{\Gamma, q_{1} \vdash_{M} m \quad \Gamma, q_{2} \vdash_{M} m}{\Gamma, q_{1} \vee q_{2} \vdash_{M} m} \vee M L$ & \\
$\frac{\Gamma_{Q} \vdash_{Q} q_{2} \Gamma, q_{1} \vdash_{M} m}{\Gamma, q_{1} / q_{2}, \Gamma_{Q} \vdash_{M} m} / M L$ & $\frac{\Gamma_{Q} \vdash_{Q} q_{1} \quad \Gamma, q_{2} \vdash_{M} m}{\Gamma, \Gamma_{Q}, q_{1} \backslash q_{2} \vdash_{M} m} \backslash M L$ \\
$\frac{\Gamma, q_{1}, \Gamma^{\prime} \vdash_{M} m}{\Gamma, q_{1} \wedge q_{2}, \Gamma^{\prime} \vdash_{M} m} \wedge M L 1$ & $\frac{\Gamma, q_{2}, \Gamma^{\prime} \vdash_{M} m}{\Gamma, q_{1} \wedge q_{2}, \Gamma^{\prime} \vdash_{M} m} \wedge M L 2$ \\
\hline
\end{tabular}

Note on the cut rules. We have two cut rules in our system: a $Q C$ ut for the $Q$-system and an $M C u t$ for the $M$-system. Although the $Q$-system subsumes a quantale logic for which cut is eliminable, for example non-commutative Linear Logic or Lambek-calculus, the $Q C u t$ of our system does not inherit this property. This is because of the modal part of the logic, that is the quantale endomorphisms and their interaction with the non-commutative sequential composition eq. (1). This equation is encoded in the $\bullet R$ rules, which needs context splitting for actions and context sharing for agents. The problem shows itself, for example, while trying to push up the cut with $f_{A}^{Q}\left(q \bullet q^{\prime}\right)$. The $M C u t$ is only eliminable in the $M$-system without the mixed rules since there is no direct interaction between conjunction and appearance maps (cf. $f_{A}^{M}$ is only join preserving) and it can be inherited from a lattice logic. Adding the mixed rules to the $M$-system creates a similar problem to the one in the $Q$-systems - e.g. try to eliminate the cut with $f_{A}^{M}(m, q)$. The problem with this cut lies in the update inequality eq. (2) and its corresponding $R$ rule where the proposition and action contexts $\Gamma$ and $\Gamma_{Q}$ are being split but the agent context $\Gamma_{A}$ is shared. Studying these issues constitutes future work.

Note on intuitive reading of sequents. To provide the reader with a way to read our sequents in natural language, we capture the intuitive meaning of a sequent in the following inductive manner:

- $\vdash_{M} m$ means "proposition $m$ holds in all contexts"

- $\vdash_{Q} q$ means "action $q$ does not necessarily have an effect on propositions"

- $\Gamma, A, \Gamma^{\prime} \vdash_{M} \delta$ means "in context $\Gamma$ agent $A$ knows/believes, that $\Gamma^{\prime} \vdash_{M} \delta$ holds" — this captures features of $A$ 's own reasoning: $\Gamma^{\prime} \vdash_{M} \delta$ is accepted by $A$ in context $\Gamma$ as a valid argument.

- $\Gamma, q, \Gamma^{\prime} \vdash_{M} \delta$ means "after action $q$ happens on context $\Gamma$, the sequent $\Gamma^{\prime} \vdash_{M} \delta$ will hold" 
- $m, \Gamma \vdash_{M} \delta$ means "in context $m$ (in any situation in which $m$ is true), the sequent $\Gamma \vdash_{M} \delta$ holds"

- $\Gamma, A, \Gamma^{\prime} \vdash_{Q} \delta$ means "in context $\Gamma$ agent $A$ knows/believes, that $\Gamma^{\prime} \vdash_{Q} \delta$ holds"

- $q, \Gamma \vdash_{Q} \delta$ means "after doing action $q$, the sequent $\Gamma \vdash_{Q} \delta$ holds"

Observe that the left-to-right order of this intuitive reading is the opposite of the right-to-left application order of $\odot$ or comma. This is because the reading involves the (intuitive) notions of knowledge and weakest precondition, which are adjoints of the $f_{A}$ and - operations; thus, the intuitive reading can be obtained by taking the adjoints (which live on the right-side of turnstile) of the formulas on the left-hand side of a sequent. For instance, the sequent $m, A, B \vdash_{M} m^{\prime}$ after applying commas on the left would mean $f_{B}^{M}\left(f_{A}^{M}(m)\right) \leq m^{\prime}$, and after applying the adjoints would correspond to $m \leq \square_{A}^{M} \square_{B}^{M} m^{\prime}$. This has now the exact shape of its intuitive meaning which is "in context $m$ agent $A$ believes that agent $B$ believes that $m^{\prime \prime}$. Examples such as $\Gamma, m \vdash_{M} m^{\prime}$ make more sense when $M$ is a Heyting algebra. For instance, the sequent $m, A, q, B, m^{\prime} \vdash_{M} m^{\prime \prime}$ can be read as: "in context $m$, agent $A$ believes that after action $q$ agent $B$ will believe that, in context $m^{\prime}$, proposition $m^{\prime \prime}$ must hold". This reading shows that, as already mentioned in the introduction, our sequent calculus expresses two forms of resource sensitivity. One is the use-only-once form of linear logic [17] that comes from the quantale structure on epistemic actions, which we called dynamic resources. The other form deals with epistemic resources: the resources available to each agent that enable him to reason in a certain way (i.e. to infer a conclusion from some assumptions). These resources are encoded in the way the context appears to the agent in sequents, for instance $\Gamma$ in the sequent $\Gamma, A, \Gamma^{\prime} \vdash_{M} m$ is the context, and hence $f_{A}^{M}(\Gamma)$ is the resource that enables agent $A$ to do the $\Gamma^{\prime} \vdash_{M} m$ reasoning. Note that $\Gamma^{\prime} \vdash_{M} m$ might not be a valid sequent in the context $\Gamma$, but it is valid in the context given by $\Gamma$ 's appearance to agent $A$.

Theorem 4.1 (Soundness) The rules presented in this section are sound with respect to the algebraic semantics in terms of the epistemic systems of Definition 2.3.

Proof. For the soundness of the rules we have to show that derivable sequents of the $Q$ and $M$-systems are valid in an epistemic system, that is $Q$-rules are valid in the quantale part and $M$-rules (including the mixed rules) are valid in the module part. In other words, we have to prove the following for the $Q$-system

$$
\text { if } \quad \Gamma \vdash_{Q} q \quad \text { then } \quad \Gamma \models_{Q} q
$$

and a similar one for the $M$-system. The proof is done as usual, that is by showing that the rules of each system preserve validity of sequents. This can easily be verified by induction on the $\odot$ operation and applying the algebraic definitions and properties of the connectives. The full proof can be found in [36], for reasons of space we provide the reader with some examples and present the proof for the rules that show the crucial features of our system: rules for sequential composition and appearance and knowledge of actions in the quantale for the $Q$-system, and rules for epistemic update and dynamic modality for the $M$-system.

i. Soundness of sequential composition. The rules for sequential composition are

$$
\frac{\Gamma, q_{1}, q_{2}, \Gamma^{\prime} \vdash_{Q} q}{\Gamma, q_{1} \bullet q_{2}, \Gamma^{\prime} \vdash_{Q} q} \bullet L \quad \frac{\Gamma_{Q}, \Gamma_{A} \vdash_{Q} q_{1} \Gamma_{Q}^{\prime}, \Gamma_{A} \vdash_{Q} q_{2}}{\Gamma_{Q}, \Gamma_{Q}^{\prime}, \Gamma_{A} \vdash_{Q} q_{1} \bullet q_{2}} \bullet R
$$

To prove soundness, we have to show that if the sequent on the top line is valid, so is the sequent on the bottom line. Using the definition of validity, we have to show the following satisfaction statement for the left rule:

$$
\text { If } \Gamma, q_{1}, q_{2}, \Gamma^{\prime} \models_{Q} q \quad \text { then } \Gamma, q_{1} \bullet q_{2}, \Gamma^{\prime} \models_{Q} q
$$


By the definition of satisfaction in terms of $\odot_{Q}$, we have to show the following

$$
\text { If } \bigodot_{Q}\left(\Gamma, q_{1}, q_{2}, \Gamma^{\prime}\right) \leq q \text { then } \bigodot_{Q}\left(\Gamma, q_{1} \bullet q_{2}, \Gamma^{\prime}\right) \leq q
$$

This is true since the application of $\bigodot_{Q}$ to the left hand side sequences of the top and bottom sequents yields equal quantale elements, that is

$$
\bigodot_{Q}\left(\Gamma, q_{1}, q_{2}, \Gamma^{\prime}\right)=\bigodot_{Q} \Gamma \bullet q_{1} \bullet q_{2} \bullet \bigodot_{Q} \Gamma^{\prime}=\bigodot_{Q}\left(\Gamma, q_{1} \bullet q_{2}, \Gamma^{\prime}\right)
$$

For the right rule we proceed similarly and show the following satisfaction statement

$$
\text { If } \Gamma_{Q}, \Gamma_{A} \models_{Q} q_{1} \quad \text { and } \quad \Gamma_{Q}^{\prime}, \Gamma_{A} \models_{Q} q_{2} \text { then } \Gamma_{Q}, \Gamma_{Q}^{\prime}, \Gamma_{A} \models_{Q} q_{1} \bullet q_{2}
$$

which is by definition equivalent to the following $\bigodot_{Q}$ statement

$$
\text { If } \bigodot_{Q}\left(\Gamma_{Q}, \Gamma_{A}\right) \leq q_{1} \quad \text { and } \bigodot_{Q}\left(\Gamma_{Q}^{\prime}, \Gamma_{A}\right) \leq q_{2} \quad \text { then } \bigodot_{Q}\left(\Gamma_{Q}, \Gamma_{Q}^{\prime}, \Gamma_{A}\right) \leq q_{1} \bullet q_{2}
$$

We first assume that we have only one agent in our agent context, that is $\Gamma_{A}=A$ and we have to show the following

$$
\text { If } f_{A}^{Q}\left(\bigodot_{Q} \Gamma\right) \leq q_{1} \quad \text { and } \quad f_{A}^{Q}\left(\bigodot_{Q} \Gamma_{Q}^{\prime}\right) \leq q_{2} \quad \text { then } \quad f_{A}^{Q}\left(\bigodot_{Q} \Gamma_{Q} \bullet \bigodot_{Q} \Gamma_{Q}^{\prime}\right) \leq q_{1} \bullet q_{2}
$$

Assume that the precedent holds, by order-preservation of the multiplication on the quantale we can multiply both sides of these inequalities and we get

$$
f_{A}^{Q}\left(\bigodot_{Q} \Gamma\right) \bullet f_{A}^{Q}\left(\bigodot_{Q} \Gamma_{Q}^{\prime}\right) \leq q_{1} \bullet q_{2}
$$

By the relation between appearance maps and multiplication on the quantale eq.(1) we have

$$
f_{A}^{Q}\left(\bigodot_{Q} \Gamma \bullet \bigodot_{Q} \Gamma_{Q}^{\prime}\right) \leq f_{A}^{Q}\left(\bigodot_{Q} \Gamma\right) \bullet f_{A}^{Q}\left(\bigodot_{Q} \Gamma_{Q}^{\prime}\right), \quad \text { hence } \quad f_{A}^{Q}\left(\bigodot_{Q} \Gamma \bullet \bigodot_{Q} \Gamma_{Q}^{\prime}\right) \leq q_{1} \bullet q_{2} .
$$

which is exactly what we wanted to prove, that is the validity of the bottom line of the rule. If $\Gamma_{A}$ has more than one agent $\Gamma_{A}=A_{1}, \ldots, A_{n}$ then we have to show that if

$$
f_{A_{1}}^{Q}\left(f_{A_{2}}^{Q}\left(\ldots f_{A_{n}}^{Q}\left(\bigodot_{Q} \Gamma\right)\right)\right) \leq q_{1} \quad \text { and } \quad f_{A_{1}}^{Q}\left(f_{A_{2}}^{Q}\left(\ldots f_{A_{n}}^{Q}\left(\bigodot_{Q} \Gamma_{Q}^{\prime}\right)\right)\right) \leq q_{2}
$$

then

$$
f_{A_{1}}^{Q}\left(f_{A_{2}}^{Q}\left(\ldots f_{A_{n}}^{Q}\left(\bigodot_{Q} \Gamma_{Q} \bullet \bigodot_{Q} \Gamma_{Q}^{\prime}\right)\right)\right) \leq q_{1} \bullet q_{2} .
$$

The proof for this case is done similarly, except that after multiplying the two sides of the assumption by $\bullet$, we have to apply the inequality for $f_{A_{i}}^{Q}$ and the quantale multiplication $n$ times, that is once for each agent $A_{i} \in \Gamma_{A}$, starting from the innermost one $f_{A_{n}}^{Q}$ and ending with the outmost one $f_{A_{1}}^{Q}$. 
ii. Soundness of appearance and knowledge of actions. The rules for the appearance map are

$$
\frac{\Gamma_{Q}, A, \Gamma \vdash_{Q} q}{f_{A}^{Q}\left(\Gamma_{Q}\right), \Gamma \vdash_{Q} q} f_{A}^{Q} L \quad \frac{\Gamma \vdash_{Q} q}{\Gamma, A \vdash_{Q} f_{A}^{Q}(q)} f_{A}^{Q} R
$$

By using the satisfaction relation and definition of $\odot_{Q}$, soundness of the left rule follows from definition of $\odot_{Q}$ between an agent and an action since $\bigodot_{Q}\left(\Gamma_{Q}, A, \Gamma\right)$ is equal to $f_{A}^{Q}\left(\bigodot_{Q} \Gamma_{Q}\right) \bullet \bigodot_{Q} \Gamma$, for which by the top line we have $f_{A}^{Q}\left(\bigodot_{Q} \Gamma_{Q}\right) \bullet \bigodot_{Q} \Gamma \leq q$. The right rule follows by the order preservation of $f_{A}^{Q}$, that is if $\bigodot_{Q} \Gamma \leq q$ then we have $f_{A}^{Q}\left(\bigodot_{Q} \Gamma\right) \leq f_{A}^{Q}(q)$, which is the meaning of the bottom line.

The rules for knowledge on the quantale are:

$$
\frac{\Gamma_{Q}, \Gamma \vdash_{Q} q}{\square_{A}^{Q}\left(\Gamma_{Q}\right), A, \Gamma \vdash_{Q} q} \square_{A} L \quad \frac{\Gamma, A \vdash_{Q} q}{\Gamma \vdash_{Q} \square_{A}^{Q}(q)} \square_{A} R
$$

Fo the left rule assume $\bigodot_{Q} \Gamma_{Q} \bullet \bigodot_{Q} \Gamma \leq q$, and we have to show $f_{A}^{Q}\left(\square_{A}^{Q}\left(\bigodot_{Q} \Gamma_{Q}\right)\right) \bullet \bigodot_{Q} \Gamma \leq q$. By composition of adjoints on the $f_{A}^{Q}$ and $\square_{A}^{Q}$, we have $f_{A}^{Q}\left(\square_{A}^{Q}\left(\bigodot_{Q} \Gamma_{Q}\right)\right) \leq \bigodot_{Q} \Gamma_{Q}$. We multiply both sides of this by $\bigodot_{Q} \Gamma$ and we get $f_{A}^{Q}\left(\square_{A}^{Q}\left(\bigodot_{Q} \Gamma_{Q}\right)\right) \bullet \bigodot_{Q} \Gamma \leq \bigodot_{Q} \Gamma_{Q} \bullet \bigodot_{Q} \Gamma$ and this is by the top line assumption less than $q$, so we have $f_{A}^{Q}\left(\square_{A}^{Q}\left(\bigodot_{Q} \Gamma_{Q}\right)\right) \bullet \bigodot_{Q} \Gamma \leq \bigodot_{Q} \Gamma_{Q} \bullet \bigodot_{Q} \Gamma \leq q$. For the right rule our top line assumption is $f_{A}^{Q}\left(\bigodot_{Q} \Gamma\right) \leq q$ which is by adjunction equal to $\bigodot_{Q} \Gamma \leq \square_{A}^{Q}(q)$. Note that this rule is also sound on the other direction, that is the bottom line implies the top line.

iii. Soundness of epistemic update. The rules for epistemic update are

$$
\frac{m^{\prime}, q, \Gamma \vdash_{M} m}{m^{\prime} \cdot q, \Gamma \vdash_{M} m} \cdot L \quad \frac{\Gamma, \Gamma_{A} \vdash_{M} m \quad \Gamma_{Q}, \Gamma_{A} \vdash_{Q} q}{\Gamma, \Gamma_{Q}, \Gamma_{A} \vdash_{M} m \cdot q} \cdot R
$$

The soundness proofs for these rules use the definition of validity and satisfaction of $M$-sequents, which is based on the $\odot_{M}$ operation. So for the left rule we have to show the following

$$
\text { If } m^{\prime}, q, \Gamma \models_{M} m \text { then } m^{\prime} \cdot q, \Gamma \models_{M} m
$$

which is by definition equivalent to the following

$$
\text { If } \bigodot_{M}\left(m^{\prime}, q, \Gamma\right) \leq m \text { then } \bigodot_{M}\left(m^{\prime} \cdot q, \Gamma\right) \leq m
$$

This holds since $\bigodot_{M}\left(m^{\prime}, q, \Gamma\right)=\bigodot_{M}\left(m^{\prime} \cdot q, \Gamma\right)=\left(m^{\prime} \cdot q\right) \wedge \bigodot_{M} \Gamma$. Proceeding similarly, for the right rule we have to show the following

$$
\text { If } \bigodot_{M}\left(\Gamma, \Gamma_{A}\right) \leq m \text { and } \bigodot_{M}\left(\Gamma_{Q}, \Gamma_{A}\right) \leq q \text { then } \bigodot_{M}\left(\Gamma, \Gamma_{Q}, \Gamma_{A}\right) \leq m \cdot q
$$

In order to do so, we first assume that we have only one agent in our agent context, that is $\Gamma_{A}=$ $A$. By the first assumption of the top line we have $f_{A}^{M}\left(\bigodot_{M} \Gamma\right) \leq m$ and by the second assumption we have $f_{A}^{Q}\left(\bigodot_{Q} \Gamma_{Q}\right) \leq q$. Since update is order preserving, we can update both sides of these two assumption by each other and get $f_{A}^{M}\left(\bigodot_{M} \Gamma\right) \cdot f_{A}^{Q}\left(\bigodot_{Q} \Gamma_{Q}\right) \leq m \cdot q$. Now by update inequality we have $f_{A}^{M}\left(\bigodot_{M} \Gamma \cdot \bigodot_{Q} \Gamma_{Q}\right) \leq f_{A}^{M}\left(\bigodot_{M} \Gamma\right) \cdot f_{A}^{Q}\left(\bigodot_{Q} \Gamma_{Q}\right) \leq m \cdot q$, which is what we want for the bottom line and we are done. If we have more than one agent, that is $\Gamma_{A}=A_{1}, \ldots, A_{n}$, then we follow the 
same line except that we have to apply the update inequality $n$ times, starting from the innermost agent $A_{n}$ to the outmost one $A_{1}$, that is

$$
f_{A_{n}}^{M}\left(f_{A_{n-1}}^{M}\left(\ldots f_{A_{1}}^{M}\left(\bigodot_{M} \Gamma \cdot \bigodot_{Q} \Gamma_{Q}\right)\right)\right) \leq m \cdot q
$$

iv. Soundness of dynamic modality. The rules for dynamic modality are

$$
\frac{m^{\prime} \vdash_{M} m \quad q \vdash_{Q} \Gamma_{Q}}{[q] m^{\prime}, \Gamma_{Q} \vdash_{M} m} D y L \quad \frac{\Gamma, q \vdash_{M} m}{\Gamma \vdash_{M}[q] m} D y R
$$

For the left rule we start from the second assumption $q \leq \bigodot_{Q} \Gamma_{Q}$, since dynamic modality is order preserving, we apply it to both sides with the proposition $m^{\prime}$ and we get

$$
[q] m^{\prime} \leq\left[\bigodot_{Q} \Gamma_{Q}\right] m^{\prime}
$$

Update is also order preserving, so we update both sides with $\bigodot_{Q} \Gamma_{Q}$ and we get

$$
[q] m^{\prime} \cdot \bigodot_{Q} \Gamma_{Q} \leq\left[\bigodot_{Q} \Gamma_{Q}\right] m^{\prime} \cdot \bigodot_{Q} \Gamma_{Q}
$$

Now by adjunction between update and dynamic modality we have that $\left[\bigodot_{Q} \Gamma_{Q}\right] m^{\prime} \cdot \bigodot_{Q} \Gamma_{Q} \leq m^{\prime}$, which provides us with

$$
[q] m^{\prime} \cdot \bigodot_{Q} \Gamma_{Q} \leq m^{\prime}
$$

now by the first assumption of the top line we have $m^{\prime} \leq m$ and by transitivity we get

$$
[q] m^{\prime} \cdot \bigodot_{Q} \Gamma_{Q} \leq m
$$

which is exactly what we want for the bottom line. We proceed similarly for the right rule, the $\odot_{M}$ definition of the top line assumption says $\bigodot_{M} \Gamma \cdot q \leq m$, which is by adjunction equivalent to $\bigodot_{M} \Gamma \leq$ $[q] m$ and the $\odot_{M}$ definition of the bottom line. Note that this rule holds in both directions, that is bottom line implies the top line.

Theorem 4.2 (Completeness) The rules presented in this section are complete with respect to the algebraic semantics in terms of the epistemic systems of Definition 2.3

Proof. In order to show the completeness of our sequent systems with regard to their algebraic semantics, we have to show that if a sequent is valid in an epistemic system then it is provable using the rules of our $Q$ and $M$-systems. That is we have to show the following

$$
\text { if } \quad \Gamma \models_{Q} q \quad \text { then } \quad \Gamma \vdash_{Q} q, \quad \text { and } \quad \text { if } \quad \Gamma \models_{M} m \quad \text { then } \quad \Gamma \vdash_{M} m \text {. }
$$

As it is usually done for the completeness proofs, we show the contrapositive: if a sequent is not provable in the system, then it is not valid in any epistemic system, that is

$$
\text { if } \Gamma \nvdash_{Q} q \text { then } \Gamma \nvdash_{Q} q, \quad \text { and } \quad \text { if } \Gamma \nvdash_{M} m \text { then } \Gamma \nvdash_{M} m \text {. }
$$


We prove these by building an epistemic system out of the syntax of our two systems in which validity and logical consequence coincide and thus a non-derivable sequent would not be valid. We denote the equivalence relation created by logical consequence $\vdash \dashv$ as $\cong$ and construct two Lindenbaum-Tarski algebras: $M_{0}$ of equivalence classes of $M$-formulas over $\cong_{M}$ and $Q_{0}$ of equivalence classes of $Q$ formulas over $\cong_{Q}$ and define an order relation $\leq$ between them as $\vdash$ on their corresponding system. Similalry, we define all the algebraic operations of epistemic systems $\wedge, \vee, f_{A}, \square_{A}, \cdot,[], \bullet$ on the quantale and module in terms of their sequent calculus counterparts, and show that these operations are well-defined over equivalence classes of formulas by using our sequent rules. We then show that these operations satisfy the finite versions of the equations of an epistemic system, by that we mean the the same axioms but with binary joins (and meets) instead of arbitrary ones. Thus we have shown that $\left(M_{0}, Q_{0},\left\{f_{A}\right\}_{A \in \mathcal{A}}\right)$ constitutes a pre-epistemic system, one with binary joins. In order to extend our proof from this pre-epistemic system to an epistemic system (with arbitrary joins), we proceed by an ideal construction, that is we build the family of ideals over $M_{0}$ and $Q_{0}$, denoted by $M$ and $Q$, and then show that $\left(M, Q,\left\{f_{A}\right\}_{A \in \mathcal{A}}\right)$ faithfully embeds $\left(M_{0}, Q_{0},\left\{f_{A}\right\}_{A \in \mathcal{A}}\right)$. This means that a non-derivable sequent of our sequent systems will not be valid in $\left(M, Q,\left\{f_{A}\right\}_{A \in \mathcal{A}}\right)$ and we have shown this for an arbitrary epistemic systems and thus all of them are complete models of our sequent systems.

The full proof is presented in [36], for reasons of space we proceed by providing the reader with some examples. In $Q_{0}$, which is the Lindenbaum-Tarski algebra of the $Q$-system, the order is the logical consequence of $Q$-sequents $\vdash_{Q}$ and the quantale operations are defined using the syntax of $Q$-formulas. Appearance maps and knowledge on $Q_{0}$ are defined using the $f_{A}^{Q}$ maps of the $Q$-system as follows

$$
f_{A}^{Q}([q]):=\left[f_{A}^{Q}(q)\right] \quad \text { and } \quad \square_{A}^{Q}[q]=\left[\square_{A}^{Q} q\right]
$$

and have to show that these definitions are well-defined, that is

$$
\text { if } \quad\left[q_{1}\right]=\left[q_{1}^{\prime}\right] \quad \text { then } \quad\left[f_{A}^{Q}\left(q_{1}\right)\right]=\left[f_{A}^{Q}\left(q_{1}^{\prime}\right)\right], \quad \text { and } \quad \text { if } \quad\left[q_{1}\right]=\left[q_{1}^{\prime}\right] \quad \text { then } \quad\left[\square_{A}^{Q} q_{1}\right]=\left[\square_{A}^{Q} q_{1}^{\prime}\right]
$$

or in logical consequence terms

$$
\text { if } \quad q_{1} \vdash_{Q} \dashv q_{1}^{\prime} \quad \text { then } \quad f_{A}^{Q}\left(q_{1}\right) \vdash_{Q} \dashv f_{A}^{Q}\left(q_{1}^{\prime}\right), \quad \text { and } \quad \text { if } \quad q_{1} \vdash_{Q} \dashv q_{1}^{\prime} \quad \text { then } \quad \square_{A}^{Q} q_{1} \vdash_{Q} \dashv \square_{A}^{Q} q_{1}^{\prime} .
$$

The proof trees for well-definedness of appearance are as follows

$$
\begin{array}{cc}
\frac{\overline{q_{1} \vdash_{Q} q_{1}^{\prime}} \text { Ass. }}{q_{1}, A \vdash_{Q} f_{A}^{Q}\left(q_{1}^{\prime}\right)} f_{A}^{Q} R & \frac{\overline{q_{1}^{\prime} \vdash_{Q} q_{1}} \text { Ass. }}{f_{A}^{Q}\left(q_{1}\right) \vdash_{Q} f_{A}^{Q}\left(q_{1}^{\prime}\right)} f_{A}^{Q} L \\
\frac{q_{1}^{\prime}, A \vdash_{Q} f_{A}^{Q}\left(q_{1}\right)}{f_{A}^{Q}\left(q_{1}^{\prime}\right) \vdash_{Q} f_{A}^{Q}\left(q_{1}\right)} f_{A}^{Q} L
\end{array}
$$

Similarly, the proof trees for well-definedness of knowledge are

$$
\begin{array}{cc}
\overline{q_{1} \vdash_{Q} q_{1}^{\prime}} \text { Ass. } & \overline{q_{1}^{\prime} \vdash_{Q} q_{1}} \text { Ass. } \\
\frac{\square_{A}^{Q} q_{1}, A \vdash q_{1}^{\prime}}{\square_{A}^{Q} q_{1} \vdash_{Q} \square_{A}^{Q} q_{1}^{\prime}} \square_{A}^{Q} R & \frac{\square_{A}^{Q} q_{1}^{\prime}, A \vdash q_{1}}{\square} \square_{A}^{Q} R
\end{array}
$$

It remains to show that appearance and knowledge are adjoint, that is

$$
\left[f_{A}^{Q}(q)\right] \leq\left[q^{\prime}\right] \quad \text { iff } \quad[q] \leq\left[\square_{A}^{Q} q^{\prime}\right]
$$


The two proof trees for these follow

$$
\frac{\overline{q \vdash_{Q} \square_{A}^{Q} q^{\prime}} \text { Ass. } \frac{\overline{q^{\prime} \vdash_{Q} q^{\prime}} I d}{\square_{A}^{Q} q^{\prime}, A \vdash_{Q} q^{\prime}} \square_{A}^{Q} L}{\frac{q, A \vdash_{Q} q^{\prime}}{f_{A}^{Q}(q) \vdash_{Q} q^{\prime}} f_{A}^{Q} L} \quad \frac{\overline{q \vdash_{Q} q} I d}{\frac{q, A \vdash_{Q} f_{A}^{Q}(q)}{f_{A}^{Q} R} \overline{f_{A}^{Q}(q) \vdash_{Q} q^{\prime}} \text { Ass. }} \text { QCut }
$$

We now have to show that our operations satisfy the binary versions of axioms of epistemic systems. For example that the appearance maps on $Q_{0}$ preserve binary joins (instead of the arbitrary joins of a quantale), that is

$$
\left[f_{A}^{Q}\left(q_{1} \vee q_{2}\right)\right]=\left[f_{A}^{Q}\left(q_{1}\right) \vee f_{A}^{Q}\left(q_{2}\right)\right]
$$

The proof of the first direction of this equality is as follows

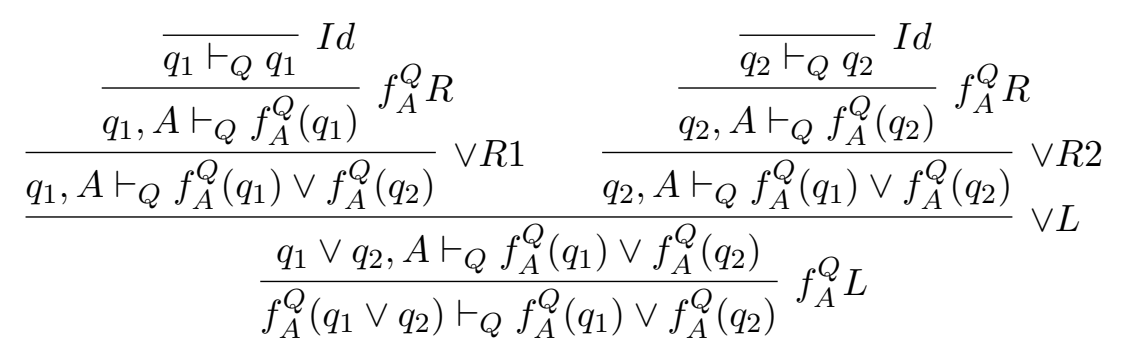

Similalry, the proof tree for the second direction is

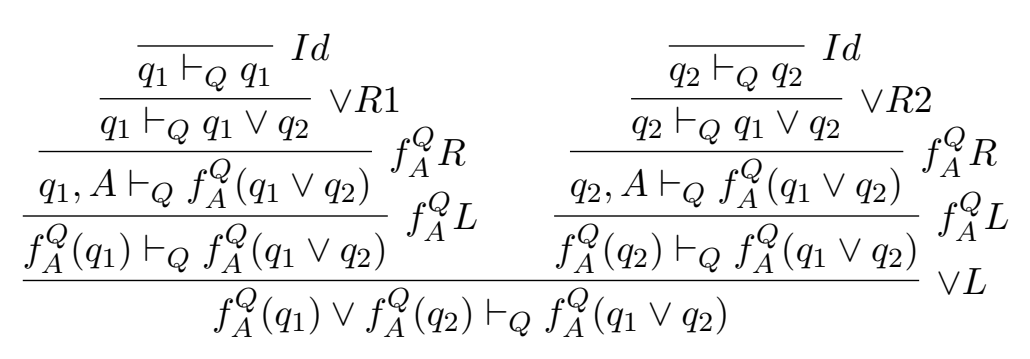

The same constructions are done in the model built out of syntax of the $M$-system, that is in $M_{0}$ where the order is $\vdash_{M}$. The meet, join, appearance and knowledge modality of $M_{0}$ are defined using their counterparts in the $M$-system, but for update and dynamic modality, we need to use both $M$ and $Q$ systems. The update is defined on the pair $\left(M_{0}, Q_{0}\right)$ using the update operator of our $M$-systems as follows

$$
[m] \cdot[q]:=[m \cdot q]
$$

We have to show that it is well-defined, that is

$$
\text { If } \quad[m]=\left[m^{\prime}\right] \text { and }[q]=\left[q^{\prime}\right] \text { then }[m \cdot q]=\left[m^{\prime} \cdot q^{\prime}\right]
$$

The proof tree for one direction of this equality is as follows

$$
\frac{\overline{m \vdash_{M} m^{\prime}} \text { Ass. } \overline{q \vdash_{Q} q^{\prime}}}{\frac{m, q \vdash_{M} m^{\prime} \cdot q^{\prime}}{m \cdot q \vdash_{M} m^{\prime} \cdot q^{\prime}} \cdot L} \cdot R
$$

The proof tree for the other direction is drawn similarly. It is easy to prove that update preserves binary joins of both $M_{0}$ and $Q_{0}$ and the unit of $Q_{0}$, and that it is associative over multiplication of $Q_{0}$. The 
dynamic modality of $M_{0}$ is defined in the same way by using the dynamic modality of the $M$-system and proved well-defined and adjoint to update.

So far we have shown that $\left(M_{0}, Q_{0},\left\{f_{A}\right\}_{A \in \mathcal{A}}\right)$ is a pre-epistemic system with regard to which $M$ and $Q$-systems are complete. We extend this proof to epistemic systems by embedding this structure into an epistemic system $\left(M, Q,\left\{f_{A}\right\}_{A \in \mathcal{A}}\right)$ by taking $M=\operatorname{Idl}\left(M_{0}\right)$ and $Q=\operatorname{Idl}\left(Q_{0}\right)$ where $\operatorname{Idl}\left(M_{0}\right)$ is the family of ideals over $M_{0}$ and $\operatorname{Idl}\left(Q_{0}\right)$ is the family of ideals over $Q_{0}$. A subset of a lattice is called an ideal if it is non-empty, downward-closed, and closed under finite joins. The order $\leq$ on ideals is given by inclusion, the arbitrary meet of ideals $\bar{\bigwedge}_{i} I_{i}$ is given by intersection of ideals $\bigcap_{i} I_{i}$, while the arbitrary join $\bar{\bigvee}_{i} I_{i}$ of a family of ideals is the ideal generated by their union, which is the downwardclosure of the set of all finite joins of elements of these ideals. For example, the join in $Q$ is given by

$$
\bar{\bigvee}_{i} I_{i}:=\downarrow\left[\left\{\bigvee Y \mid Y \text { finite } \subseteq \bigcup_{i} I_{i}\right\}\right] .
$$

The rest of operations, that is $f_{A}$ for both $M$ and $Q$, also $\cdot$ and $\bullet$ are extended to ideals by applying them pointwise and then taking the downward closure. For instance, the appearance of ideals on $Q$ is defined as follows

$$
\overline{f_{A}^{Q}}(I)=\downarrow\left[\left\{f_{A}^{Q}(q) \mid q \in I\right\}\right]
$$

We have to show that these operations are ideal preserving, that is for example, the join of ideals $\bar{\bigvee}_{i} I_{i}$ is an ideal. Downward closure follows from the definition. For closure under joins assume that $x, y \in$ $\bar{\bigvee}_{i} I_{i}$, then $x=\bigvee Y_{1}$ and $y=\bigvee Y_{2}$, for $Y_{1}, Y_{2}$ finite subsets of the unions of $I_{i}$ 's, that is $Y_{1} \subseteq I_{1}$ and $Y_{2} \subseteq I_{2}$. We have $x \vee y=\left(\bigvee Y_{1}\right) \vee\left(\bigvee Y_{2}\right)=\bigvee\left(Y_{1} \vee Y_{2}\right)$, since $Y_{1} \vee Y_{2} \subseteq I_{1} \cup I_{2}$, it is also a finite subset of union of $I_{i}$ 's and thus $x \vee y \in \bar{\bigvee}_{i} I_{i}$. The proofs for other operations are done similarly, see [36]. The units of these operations are extended to ideals, the unit of multiplication is $\downarrow 1$, the unit of appearance and join of $Q$ and $M$ is $\{\perp\}$ for the bottom of each accordingly, the unit of their meets is the ideal generated by the whole of $Q_{0}$ and $M_{0}$, that is $Q_{0}$ and $M_{0}$ themselves. These ideals satisfy the axioms of epistemic systems, for example appearance of ideals of $Q_{0}$ preserves arbitrary joins of them. These are straightforward proofs and follow from the definition, for example for appearance of ideals of $Q_{0}$ we have to show

$$
\overline{f_{A}^{Q}}\left(\bigvee_{i} I_{i}\right)=\bar{\bigvee}_{i} \overline{f_{A}^{Q}}\left(I_{i}\right)
$$

We start from the left hand side

$$
\begin{aligned}
\overline{f_{A}^{Q}}\left(\bar{\bigvee}_{i} I_{i}\right) & =\downarrow\left\{f_{A}^{Q}\left(\bar{\bigvee} I_{i}\right) \mid I_{i} \text { is an ideal }\right\} \\
& =\downarrow\left\{f_{A}^{Q}(\bigvee Y) \mid Y \text { finite } \subseteq \bigcup_{i} I_{i}\right\} \\
& =\downarrow\left\{\bigvee f_{A}^{Q}(Y) \mid Y \text { finite } \subseteq \bigcup_{i} I_{i}\right\}
\end{aligned}
$$

which is equal to $\overline{\mathrm{V}}_{i} \bar{f}_{A}^{Q}\left(I_{i}\right)$. The proofs for other axioms are done similarly and from them it follows that $\left(M, Q,\left\{f_{A}\right\}_{A \in \mathcal{A}}\right)$ is an epistemic system. It remains to show that $M_{0}$ and $Q_{0}$ are faithfully embedded into $M$ and $Q$. The embedding $Q_{0} \hookrightarrow \operatorname{Idl}\left(Q_{0}\right)$ is defined as $q \mapsto \downarrow q$, and similarly for $M_{0} \hookrightarrow \operatorname{Idl}\left(M_{0}\right)$ as $m \mapsto \downarrow m$. We show that these are homomorphisms, for example in $Q$ by checking the following for $q_{1}, q_{2} \in Q_{0}$

$$
e\left(q_{1}\right) \bigcirc e\left(q_{2}\right)=e\left(q_{1} \bigcirc q_{2}\right)
$$


for $\bigcirc \in\{\bar{\bigvee}, \bar{\bigwedge}, \bar{\bullet}\}$ and also for the appearance map as follows

$$
e\left(f_{A}^{Q}(q)\right)=\overline{f_{A}^{Q}}(e(q))
$$

For instance for the proof of appearance maps of $Q$ we have to show $\overline{f_{A}^{Q}}(\downarrow q)=\downarrow f_{A}^{Q}(q)$, which is equivalent to the following by definition of appearance of ideals

$$
\downarrow f_{A}^{Q}(q)=\overline{f_{A}^{Q}}(\downarrow q)=\downarrow\left\{f_{A}^{Q}(x) \mid \forall x \in \downarrow q\right\}
$$

For the first direction we take an element of the left hand side $x \leq f_{A}^{Q}(q)$ and since $q \leq q$, we have $f_{A}^{Q}(q) \in \overline{f_{A}^{Q}}(\downarrow q)$ and we get $x \in \overline{f_{A}^{Q}}(\downarrow q)$. For the other direction we take an element of the right hand side $x \in \overline{f_{A}^{Q}}(\downarrow q)$, which means $x \leq f_{A}^{Q}(y)$ for some $y \leq q$. Since $f_{A}^{Q}$ is monotone, we take apply it to both sides of $y \leq q$ and we get $f_{A}^{Q}(y) \leq f_{A}^{Q}(q)$, so we have that $x \leq f_{A}^{Q}(q)$, that is an element of the left hand side.

Since the pre-epistemic system $\left(M_{0}, Q_{0},\left\{f_{A}\right\}_{A \in \mathcal{A}}\right)$ with binary operations was a complete model of our systems and the embedding is faithful, the epistemic system remains a complete model of our $M$ and $Q$-systems, that is every non-derivable sequent of our systems will be invalid in $\left(M, Q,\left\{f_{A}\right\}_{A \in \mathcal{A}}\right)$.

Example of a derivation. We prove a derived rule that allows us to predict knowledge of agents after an action based on their appearance of the action and by permuting the dynamic and epistemic modalities ${ }^{3}$ :

\section{Action-Knowledge Lemma.}

$$
\square_{A}\left[f_{A}^{Q}(q)\right] m \vdash[q] \square_{A} m
$$

Proof. The proof tree is as follows

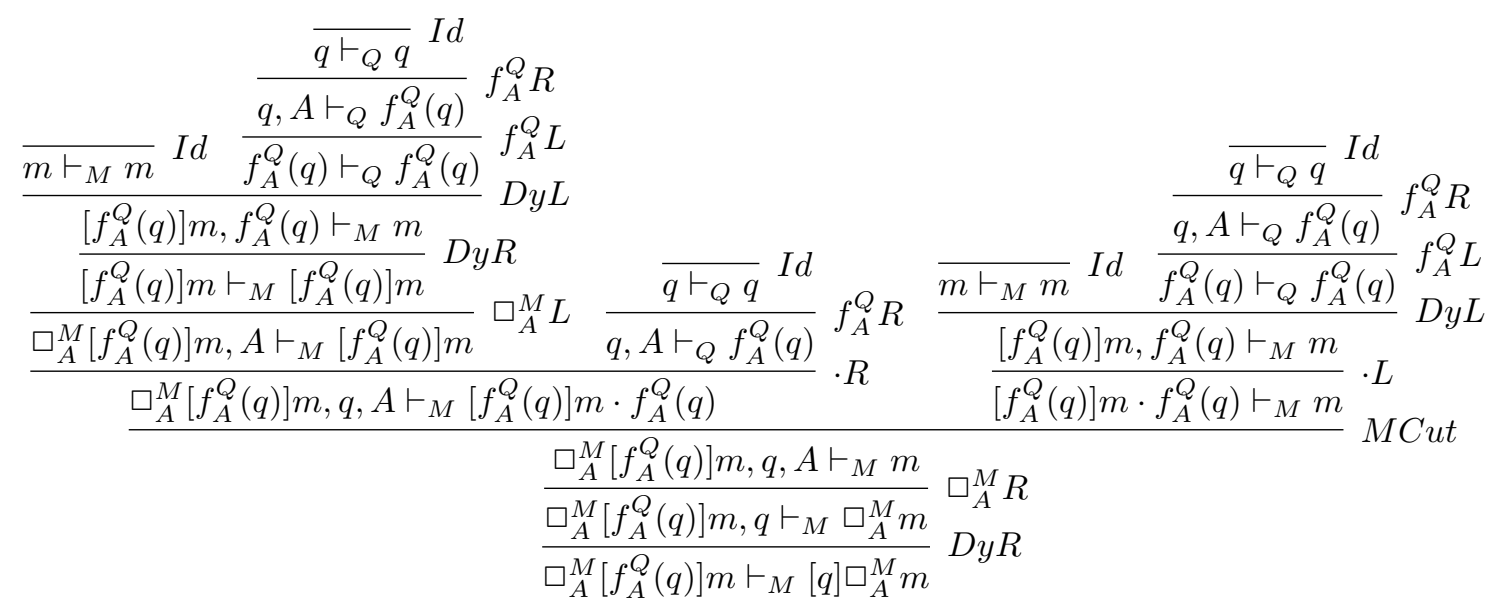

\footnotetext{
${ }^{3}$ This rule corresponds to a non-Boolean version of the so-called "Action-Knowledge Axiom" of BMS [6].
} 
Example of an application. We present the proof tree of the property that we proved for the MITM cryptographic attack in the algebra section. In order to encode the scenario in the sequent calculus, we have to add axioms for our appearances, facts, and kernel assumptions. For the appearance of propositions we have the following axiom schema for the $M$-systems (we refer to all these assumption axioms as Ass.):

$$
\overline{m, A \vdash_{M} m^{\prime}} \text { Ass. } \quad \text { iff } \quad f_{A}^{M}(m)=m^{\prime}
$$

Similarly, the following is the axiom schema for the appearance of actions in the $Q$-system

$$
\overline{q, A \vdash_{M} q^{\prime}} \text { Ass. } \quad \text { iff } \quad f_{A}^{Q}(q)=q^{\prime}
$$

For the kernel of actions, we have the following schema

$$
\overline{m, q \vdash_{M} \perp} \text { Ass. } \quad \text { iff } \quad m=\operatorname{Ker}(q)
$$

and finally we encode the entailment between propositions and facts $m \leq \varphi$ via the following schema

$$
\overline{m \vdash_{M} \varphi} \text { Ass. } \quad \text { iff } \quad m \leq \varphi
$$

We encode the cryptographic attack scenario by instantiating these axioms. The axioms for the facts $P, \bar{P}$ and propositions $s, t$ will be the following

$$
\overline{s \vdash_{M} P} \text { Ass. } \quad \overline{t \vdash_{M} \bar{P}} \text { Ass. }
$$

We considered the kernel of four actions $\left\{\alpha, \alpha^{\prime}, \beta, \beta^{\prime}\right\}$ encoded as follows

$$
\overline{\bar{P}, \alpha \vdash_{M} \perp} \text { Ass. } \overline{\bar{P}, \alpha^{\prime} \vdash_{M} \perp} \text { Ass. } \overline{P, \beta \vdash_{M} \perp} \text { Ass. } \overline{P, \beta^{\prime} \vdash_{M} \perp} \text { Ass. }
$$

The encoding of the appearances of the propositions and actions to our three agents $\{A, B, C\}$ is straightforward, for example the ones used in the proof are encoded as follows on the $M$-system

$$
\overline{s, A \vdash_{M} s} \text { Ass. } \overline{s, B \vdash_{M} s \vee t} \text { Ass. }
$$

and as follows for the actions in the $Q$-system

$$
\overline{\alpha, A \vdash_{Q} \alpha^{\prime}} \text { Ass. } \overline{\alpha, B \vdash_{Q} \beta^{\prime}} \text { Ass. } \quad \overline{\alpha^{\prime}, B \vdash_{Q} \beta^{\prime}} \text { Ass. }
$$

We prove that in the real state $s$ and after communicating the secret $P$ or $\bar{P}$ via the action $\alpha \vee \beta$, agent $A$ knows that $B$ knows that $P$ holds, that is $s \cdot(\alpha \vee \beta) \vdash_{M} \square_{A}^{M} \square_{B}^{M} P$. One crucial part of the proof is cut with an update formula and then the application of the left and right update rules to reduce the update to the assumptions axioms. The trick is to cut a sequent that looks like $m, q, A \vdash_{M} m^{\prime \prime}$ with an update formula $m^{\prime} \cdot q^{\prime}$ the proposition part of which is the appearance of the proposition on the left hand side, that is $f_{A}^{M}(m)=m^{\prime}$, and the action part of which is the appearance of the action on the left hand side, that is $f_{A}^{Q}(q)=q^{\prime}$. The other important part of the proof is cutting with $\perp$ and using the kernel assumption axioms. The steps of the proof are more or less the same as in the algebra. The proof tree of is as follows (in order to fit it in the page we had to draw two of its sub-trees $\Pi 1$ and $\Pi 2$ separately) 


$$
\begin{aligned}
& \frac{\overline{s, B \vdash_{M} s \vee t} \text { Ass. } \quad \frac{\overline{\alpha^{\prime}, B \vdash_{Q} \alpha^{\prime}}}{A s s .} \cdot R \quad \frac{\Pi 1}{\frac{s, \alpha^{\prime}, B \vdash_{M}(s \vee t) \cdot \alpha^{\prime}}{s, \alpha^{\prime}, B \vdash_{M} P}} \cdot L}{(s \vee t) \cdot \alpha^{\prime} \vdash_{M} P} \cdot M C u t \\
& \begin{array}{cll}
\overline{s, A \vdash_{M} s} \text { Ass. } \quad \overline{\alpha, A \vdash_{Q} \alpha^{\prime}} \text { Ass. } & \frac{s, \alpha^{\prime}, B \vdash_{M} P}{s, \alpha^{\prime} \vdash_{M} \square_{B}^{M} P} \square_{B}^{M} R \\
\frac{s, \alpha, A \vdash_{M} s \cdot \alpha^{\prime}}{s \cdot \alpha^{\prime} \vdash_{M} \square_{B}^{M} P} \cdot L \\
M C u t
\end{array} \\
& \frac{\frac{s, \alpha, A \vdash_{M} \square_{B}^{M} P}{s, \alpha \vdash_{M} \square_{A}^{M} \square_{B}^{M} P} \square_{A}^{M} R}{\frac{s, \alpha \vee \beta \vdash_{M} \square_{A}^{M} \square_{B}^{M} P}{s \cdot(\alpha \vee \beta) \vdash_{M} \square_{A}^{M} \square_{B}^{M} P} \cdot L}
\end{aligned}
$$

The sub-proof trees $\Pi 1$ and $\Pi 2$ are below

$\Pi 1:$

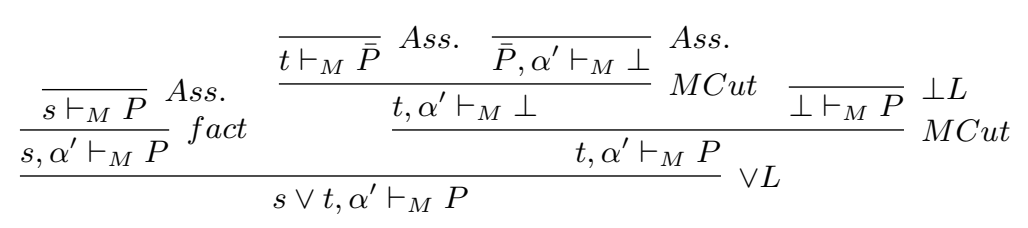

П2:

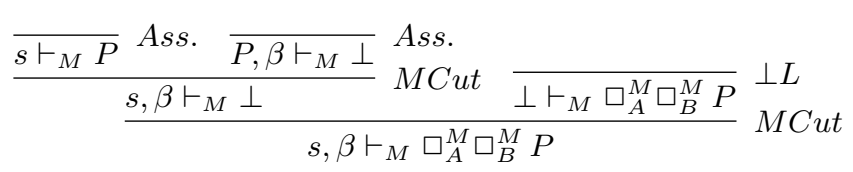

\section{Conclusion and further elaborations}

We have developed an algebraic axiomatics in terms of a simple mathematical object: a sup-lattice $M$, which encodes epistemic propositions and facts; a quantale $Q$ (acting on $M$ ) which encodes epistemic actions (and the updates induced by them); and a family of (lax-)endomorphisms of the structure $\left(M, Q, \bigvee_{M}, \bigvee_{Q}, \cdot, \bullet, 1\right)$, encoding the agents' information states. From this structure many useful other modalities arise, including dynamic modalities, epistemic modalities and residuals. This algebraic axiomatics is a dynamic epistemic logic and generalizes the BMS logic of [6] to non-Boolean settings, while capturing the same concepts, and enriches it with a logical account of dynamic and epistemic resources in terms of actions and agents. We have presented a sound and complete sequent calculus that enables us to deal with dynamic epistemic scenarios using semi-automatic proof techniques. As examples of application, we have encoded and analyzed a classic epistemic puzzle (Muddy Children) and some of new variations of it with lying and cheating children, and proved the correctness of a simple security protocol, both algebraically and by a proof in sequent calculus. Some possible further elaborations on this line of thought follow.

- In this paper, following dynamic epistemic logic, we dealt with the same update schema for all agents. This is a postulate of "uniform rationality" and it means that the mechanism for information update is the same for all agents. It makes sense, if not being necessary, to consider personalized updates, where each agent updates his information in a different way than other agents do. We think that such personalized updates could be better dealt with by moving to a categorical semantics. More explicitly, we are working in an enriched categorical setting where a 
quantale $Q$ is a one-object quantaloid, i.e. a one-object sup-enriched category, and agents' personalized updates $\mathcal{M}_{A}$ are sup-enriched functors. Appearance maps arise as lax sup-enriched natural transformations between the update functors. It would be interesting to compare our categorical approach with coalgebraic epistemic features which are currently studied e.g. [2].

- The Kripke semantics of a dynamic epistemic logic has been used as an alternative to BAN logic to reason about security protocols e.g. in [22]. As shown in [36] ch. 5, our algebraic setting provides an elegant frame work that facilitates these security applications. We would like to extend the domain of such applications to be able to encode and prove the correctness of open security protocols, for example by adding more types to our setting through a quantaloid enrichment [37].

- Approximation and probability. We can conceive the modules in our setting as a more general type of partial orders than merely an algebraic logic. We can accommodate additional computational structure e.g. a domain structure [13], quantitative valuations of content [19, 29], or a combination of these which enables accommodating probabilities e.g. the partial order on probability measures introduced in [9] is defined in terms of a Bayesian update operation. This development would also be help in application to security.

- Part of the motivation of this work was a marriage of epistemics and resource-sensitivity [28]. Although we have introduced dynamic and epistemic resources in our setting, we would like to refine our logic and make it more resource-sensitive by relativizing our notion of "consequence" to "logical" actions available to agents. This will allow us to deal with classical resource sensitive problems such as the problem of logical omniscience. The two examples below might provide useful insights, fragments and tools. (i) In the money games of [24] the resource, i.e., money $x \in \mathbb{R}^{+}$, is encoded using the quantale structure of $\mathbb{R}^{+}$as a base for enrichment. The underlying lattices are free lattices which adds linearity to the propositions. They moreover admit a game-theoretic interpretation [24]. (ii) The logic of bunched implications of [32] also provides a model to to handle resources which freely combines intuitionistic additive and multiplicative linear structure via contexts. The semantics in terms of Grothendieck sheaves of the additives again indicates a monoid-enriched structure in the sense of [37].

- We would like to eliminates cuts from this system, for which as suggested by our referee, a deep inference deductive system in the calculus of structures might be one way. We would also like to develop a boolean version of the sequent calculus presented here for concrete epistemic systems and prove its completeness with regard to Kripke semantics. Such a development will lead to a more refined version of our representation Theorem 3.3 for a boolean dynamic epistemic logic.

\section{Acknowledgements}

We thank Samson Abramsky, André Joyal, Dusko Pavlovic, Greg Restall, and Isar Stubbe for valuable discussions, and our referee Lutz Straßburger for his useful detailed comments and corrections on the first version of this paper, and for his suggestion for the presentation of the sequent calculus. M. S. thanks Samson Abramsky and Oxford University Computer Laboratory for their hospitality, Mathieu Marion for his logistic support, and Roy Dyckhoff for his comments on cut-elimination. B. C. is supported by the EPSRC grant EP/C500032/1 High-Level Methods in Quantum Computation and Quantum Information. 


\section{References}

[1] S. Abramsky and S. Vickers. 'Quantales, observational logic and process semantics'. Mathematical Structures in Computer Science 3, 161-227, 1993.

[2] A. Baltag. 'A coalgebraic semantics for epistemic programs'. In: Proceedings of Coalgebraic Methods in Computer Science'03, 2003.

[3] A. Baltag. 'A Logic for Suspicious Players: Epistemic Actions and Belief Updates in Games'. Bulletin of Economic Research 54, 1-46, 2002.

[4] A. Baltag, B. Coecke and M. Sadrzadeh. 'Algebra and Sequent Calculus for Epistemic Action'. In Electrical Notes in Computer Science 126, pp. 27-52, 2005.

[5] A. Baltag and L.S. Moss. 'Logics for epistemic programs'. Synthese 139, 2004.

[6] A. Baltag, L.S. Moss and S. Solecki. 'The logic of public announcements, common knowledge and private suspicions'. CWI Technical Report SEN-R9922, 1999.

[7] A. Baltag and M. Sadrzadeh. 'The Algebra of Multi-Agent Dynamic Belief Revision'. To appear in Electronic Notes in Theoretical Computer Science, proceedings of 3rd workshop on Logic and Communication in Multi-Agent Systems in IJCAI, Edinburgh, 2005.

[8] J. Barwise, D. Gabbay and C. Hartonas. 'On the logic of information flow'. Bulletin of the Interest Group in Pure and Applied Logics 3, 7-49, 1995.

[9] B. Coecke and K. Martin. A Partial Order on Classical and Quantum States. Research Report PRG-RR-02-07, Oxford University Computing Laboratory, 2002. http://web.comlab.ox.ac.uk/oucl/publications/tr/rr-02-07.html

[10] B. Coecke, D. J. Moore and I. Stubbe. 'Quantaloids describing causation and propagation of physical properties'. Foundations of Physics Letters 14, 133-145, 2001.

[11] E.W. Dijkstra. A Discipline of Programming. Prentice-Hall, 1976.

[12] R. Fagin, J. Y. Halpern, Y. Moses and M. Y. Vardi. Reasoning about Knowledge. MIT Press, 1995.

[13] G. Gierz, K. H. Hofmann, K. Keimel, J. D. Lawson, M. W. Mislove and D. S. Scott. A Compendium of Continuous Lattices. Springer-Verlag, 1980.

[14] J. Gerbrandy. 'Dynamic Epistemic Logic'. In L.S. Moss, et al (eds.) Logic, Language, and Information 2, Stanford University, CSLI Publication, 1999.

[15] J. Gerbrandy. Bisimulation on Planet Kripke. Ph.D. dissertation, University of Amesterdam, 1999.

[16] J. Gerbrandy, and W. Groenveld. 'Reasoning about information change'. Journal of Logic, Language, and Information 6, 1997.

[17] J-Y. Girard. 'Linear logic'. Theoretical Computer Science 50,1-102, 1987.

[18] B. von Karger, 'Temporal Algebras', Math. Struc. in Comp. Sci. 8, pp. 277-320, 1998.

[19] R. Kopperman, S. Matthews and H. Pajoohesh. 'Partial metrizability in value quantales'. preprint, 2004.

[20] D. Harel, D. Kozen and J. Tiuryn. Dynamic Logic. MIT Press, 2000.

[21] C. A. R. Hoare and Jifeng, HE. 'The weakest prespecification'. Information Processing Letters 24, 127-132, 1987.

[22] A. Hommersom, J.J. Meyer and E. De Vink. 'Update Semantics of Security Protocols'. Synthese 142, 289-327, 2004.

[23] P. T. Johnstone. Stone Spaces. Cambridge University Press, 1982. 
[24] A. Joyal. 'Free lattices, communication and money games'. In: M. L. Dalla Chiara et al. (eds.), Logic and Scientific Methods, Kluwer, 29-68, 1997.

[25] A. Joyal and M. Tierney. 'An extension of the Galois theory of Grothendieck'. Memoirs of the American Mathematical Society 309, 1984.

[26] F. Lamarche and C. Retoré. 'Proof Nets for the Lambek Calculus - An Overview'. V.M. Abrusci and C. Casadia (eds.), Proceedings of the 1996 Workshop on Proofs and Linguistic Categories, CLUEB, pp. 241-262, Roma, April 1996.

[27] J. Lambek. 'The mathematics of sentence structure'. American Mathematics Monthly 65, 154-169, 1958.

[28] M. Marion and M. Sadrzadeh. 'Reasoning about knowledge in linear logic: modalities and complexity’. In: D. Gabbay, S. Rahman, J. M. Torres and J.-P. Van Bendegem (eds.), Logic, Epistemology, and the Unity of Science, Kluwer, 2004.

[29] K. Martin. A Foundation for Computation. Ph.D. Thesis, Tulane University, 2000.

[30] C. J. Mulvey. \&. Supplemento ai Rendiconti del Circolo Matematico di Palermo II, 99-104, 1992.

[31] J. Plaza. 'Logics of public communications'. Proceedings of 4th International Symposium on Methodologies for Intelligent Systems, 1989.

[32] P. W. O'Hearn and D. J. Pym. 'The logic of bunched implications'. Bulletin of Symbolic Logic 5, 215-244, 1999.

[33] J. Paseka and J. Rosicý. 'Quantales'. In: B. Coecke, D. J. Moore and A. Wilce (eds.), Current Research in Operational Quantum Logic: Algebras, Categories, Languages, Kluwer, 245-262, 2000.

[34] P. Resende. 'Quantales and observational semantics'. In: B. Coecke, D. J. Moore and A. Wilce (eds.), Current Research in Operational Quantum Logic: Algebras, Categories, Languages, Kluwer, 263-288, 2000.

[35] K. I. Rosenthal. Quantales and their Applications. Pitman Research Notes in Mathematics Series 234, Longman, 1990.

[36] M. Sadrzadeh. Actions and Resources in Epistemic Logic. Ph.D. Thesis, Université du Québec À Montréal. http://www.ecs. soton.ac.uk/ ms6/all.pdf

[37] I. Stubbe. Categorical Structures Enriched in a Quantaloid: Categories and Semicategories. Ph.D. Thesis, Université Catholique de Louvain, 2003.

[38] J. Van Benthem. 'Logic in action'. Journal of Philosophical Logic 20, 225-263, 1989.

[39] F. Wolter and M. Zakharyaschev. 'The relation between intuitionistic and classical modal logics'. Algebra and logic 36, 73-92, 1997.

[40] D.N. Yetter. 'Quantales and (non-commutative) Linear Logic'. Journal of Symbolic Logic 55, 4164, 1990. 